LPENSL-TH-03/10

DESY 11-037

\title{
Thermodynamic limit of particle-hole form factors in the massless XXZ Heisenberg chain
}

\author{
N. Kitanine 1 , K. K. Kozlowski 2 , J. M. Maillet ${ }^{3}$, \\ N. A. Slavnov4, V. Terras
}

\begin{abstract}
We study the thermodynamic limit of the particle-hole form factors of the XXZ Heisenberg chain in the massless regime. We show that, in this limit, such form factors decrease as an explicitly computed power-law in the systemsize. Moreover, the corresponding amplitudes can be obtained as a product of a "smooth" and a "discrete" part: the former depends continuously on the rapidities of the particles and holes, whereas the latter has an additional explicit dependence on the set of integer numbers that label each excited state in the associated logarithmic Bethe equations. We also show that special form factors corresponding to zero-energy excitations lying on the Fermi surface decrease as a power-law in the system size with the same critical exponents as in the longdistance asymptotic behavior of the related two-point correlation functions. The methods we develop in this article are rather general and can be applied to other massless integrable models associated to the six-vertex $R$-matrix and having determinant representations for their form factors.
\end{abstract}

\section{Introduction}

This article is a continuation of our study of form factors in massless quantum integrable models. We have recently [1] investigated the thermodynamic limit of a special, Umklapptype, form factor of the $X X Z$ Heisenberg spin chain [2, 3, 4, 5, 6, 7, 8, 9] starting from its

\footnotetext{
${ }^{1}$ IMB, UMR 5584 du CNRS, Université de Bourgogne, France, Nicolai.Kitanine@u-bourgogne.fr

${ }^{2}$ DESY, Hamburg, Deutschland, karol.kajetan.kozlowski@desy.de

${ }^{3}$ Laboratoire de Physique, UMR 5672 du CNRS, ENS Lyon, France, maillet@ens-lyon.fr

${ }^{4}$ Steklov Mathematical Institute, Moscow, Russia, nslavnov@mi.ras.ru

${ }^{5}$ Laboratoire de Physique, UMR 5672 du CNRS, ENS Lyon, France, veronique.terras@ens-lyon.fr
} 
determinant representation [10] in the algebraic Bethe Ansatz framework [11, 12, 13]. We now apply this method to general, particle-hole type, form factors. The main goal of our analysis is to pave a way for the calculation of the asymptotic behavior of correlation functions in massless integrable models through their form factor expansion. Our results should also be useful in the numerical computation of dynamical structure factors through their (exact) form factor expressions starting from finite size systems [14, 15, 16, 17, 18] as they allow a precise a priori control of the dependence of each form factor in terms of the system size.

For definiteness, we chose to focus on a particular model, the XXZ spin-1/2 Heisenberg chain [2] in the massless regime and in an external magnetic field $h>0$ for which determinant representations of general form factors were obtained in [10]. However, up to minor modifications specific to the choice of the model, our method and results apply as well to other massless, algebraic Bethe Ansatz-solvable models with known determinant representations of their form factors like the non-linear Schrödinger model [19, 20] or the higher spin XXX [21] and XXZ 22. chains.

The Hamiltonian of the XXZ chain is given by,

$$
H=\sum_{k=1}^{M}\left(\sigma_{k}^{x} \sigma_{k+1}^{x}+\sigma_{k}^{y} \sigma_{k+1}^{y}+\Delta\left(\sigma_{k}^{z} \sigma_{k+1}^{z}-1\right)\right)-\frac{h}{2} \sum_{k=1}^{M} \sigma_{k}^{z},
$$

where $\sigma_{k}^{x, y, z}$ are the spin operators (Pauli matrices) acting on the $k^{\text {th }}$ site of the chain. The main purpose of this article is to study the asymptotic behavior of the form factors in the thermodynamic limit $M \rightarrow \infty$ of the chain and in the massless regime $|\Delta|<1$. We will take this limit starting from a chain of finite even size and subject to periodic boundary conditions.

Considering the finite chain allows us to define rigorously the form factors of local spin operators as the normalized matrix elements

$$
\mathcal{F}_{\psi \psi^{\prime}}^{(s)}(m)=\frac{\left\langle\psi\left|\sigma_{m}^{s}\right| \psi^{\prime}\right\rangle}{\|\psi\| \cdot\left\|\psi^{\prime}\right\|}, \quad s=x, y, z
$$

where $|\psi\rangle$ and $\left|\psi^{\prime}\right\rangle$ are two eigenstates of the Hamiltonian (1.1). Such matrix elements can be computed in a systematic way by using the solution to the quantum inverse scattering problem [10, 23] together with the explicit formulae for scalar products [24, 25, 10] in the algebraic Bethe Ansatz framework [11, 12, 13, 26].

Using the closure relation, one can express any zero temperature two-point correlation function of spin operators as a sum over the form factors of the corresponding local operators,

$$
\frac{\left\langle\psi_{g}\left|\sigma_{m}^{s} \sigma_{m^{\prime}}^{s^{\prime}}\right| \psi_{g}\right\rangle}{\left\langle\psi_{g} \mid \psi_{g}\right\rangle}=\sum_{\left|\psi^{\prime}\right\rangle} \mathcal{F}_{\psi_{g} \psi^{\prime}}^{(s)}(m) \mathcal{F}_{\psi^{\prime} \psi_{g}}^{\left(s^{\prime}\right)}\left(m^{\prime}\right)
$$

Here $\left|\psi_{g}\right\rangle$ denotes the ground state of the Hamiltonian (1.1), and the summation is taken with respect to all the eigenstates $\left|\psi^{\prime}\right\rangle$ of (1.1).

One possible way of dealing with (1.3) was proposed in [27, 28, where the sum over the form factors for the finite chain was recast into a multiple contour integral, the so-called master equation. This method then leads to various multiple integral representations for the correlation functions in the thermodynamic limit $[27,28,29]$ that are in fact non-trivial summations of 
previously obtained multiple integral representations for the correlation functions [30, 31, 32, 33, 34, 35]. Moreover, in [36] we have shown how to derive long-distance asymptotic behavior of certain two-point correlation functions using such master equation representation. It agrees with predictions arising from the Luttinger-liquid and Conformal Field Theories approaches [37, 38, 39, 40, 41, 42, 43, 44]. It is the very remarkable structure of the results obtained in [36] (the amplitudes of the power law decrease of the long distance behavior of the two-point correlation function are related to the modulus squared of some properly normalized form factors) that strongly suggested that another possible way to analyze the asymptotic behavior of the two-point correlation functions would be to take the thermodynamic limit directly in (1.3). It seems indeed quite natural that, in this limit, the main contribution to the sum (1.3) should stem from excitations above the ground state having a finite energy. Such excited states can be characterized in terms of particles, holes and/or string configurations. In this framework, the sum over the complete set of states in (1.3) becomes a sum over all the possible particlehole-string type excitations above the ground state which, after some possible regularizations, should be replaceable by integrals in the thermodynamic limit. Another important motivation to learn how to deal directly with the form factor expansion of the correlation functions concerns the time dependent case, as in that situation the asymptotic analysis through the dynamical master equation [28] poses yet unsolved problems. The first step to carry out this program is therefore to determine the leading asymptotic behavior of the form factors of the model in the thermodynamic limit. It is the purpose of the present article to solve this problem for the so-called particle/hole type form factors (corresponding to diffusion states). The case of states associated with complex solutions of the Bethe equations such as string type solutions (corresponding to bound states) requires the use of additional techniques and will be considered in a separate publication.

In [1], we have shown that, in the $M \rightarrow \infty$ limit, a special Umklapp-type form factor of the $\sigma^{z}$ operator decreases as some negative power of the system size $M$. The striking feature of this analysis is that this power coincides with one of the critical exponents appearing in the longdistance asymptotic behavior of the zero temperature two-point correlation function $\left\langle\sigma_{1}^{z} \sigma_{m+1}^{z}\right\rangle$. In the present article, we will show that general form factors exhibit similar properties: they all decay as some negative power of the size $M$; moreover, for special types of form factors corresponding, in the thermodynamic limit, to particle-hole excitations lying on the Fermi surface, these exponents coincide exactly with the critical exponents describing the powerlaw decay of the long-distance asymptotic behavior of the corresponding two-point correlation functions.

More precisely, in the thermodynamic limit, the product of two form factors appearing in (1.3) can be presented in the following form

$$
\mathcal{F}_{\psi_{g} \psi^{\prime}}^{(s)}\left(m^{\prime}\right) \cdot \mathcal{F}_{\psi^{\prime} \psi_{g}}^{\left(s^{\prime}\right)}(m)=M^{-\theta_{s s^{\prime}}} e^{i \mathcal{P}_{e x}\left(m-m^{\prime}\right)} \mathcal{S}_{s s^{\prime}} \mathcal{D}_{s s^{\prime}}
$$

All the dependence on the lattice distance $m-m^{\prime}$ is contained in an obvious phase factor 1 . There $\mathcal{P}_{\text {ex }}$ is the excitation momentum. The finite part of the form factors product can be separated into two parts $\mathcal{S}_{s s^{\prime}}$ and $\mathcal{D}_{s s^{\prime}}$. We call them smooth and discrete parts respectively.

\footnotetext{
${ }^{1}$ Some form factors (see e.g. (3.1) ) may also have an additional factor $(-1)^{m-m^{\prime}}$, which can be removed by the re-definition of the Hamiltonian (1.1)
} 
We show that the smooth part $\mathcal{S}_{s s^{\prime}}$ depends continuously on the rapidities of the particles and holes. On the contrary, it is only when the particles (holes) rapidities are separated from the Fermi boundary that the discrete part $\mathcal{D}_{s s^{\prime}}$ does also depend smoothly on these quantities. As soon as the latter approach the Fermi surface, the discrete structure of the form factors reveals itself in $\mathcal{D}_{s s^{\prime}}$. This means that a microscopic (of order $1 / M$ ) deviation of a particle (hole)'s rapidity leads to a macroscopic change of $\mathcal{D}_{s s^{\prime}}$. We believe that one of the consequences of such a structure is the particular role played by the Fermi boundary in bosonization techniques.

The article is organized as follows. In section 2 we give a description of the low-lying excited states of the model. Following the ideas of [1], we consider excitations above the ground state of the twisted transfer matrix. This enables us to formulate precisely our results in section 3 , i.e. the structure of the large size asymptotic behavior of general particle-hole form factors in the XXZ spin-1/2 chain. The remaining part of the article is devoted to the proof of this result. In section 4 we show how form factors can be reduced to scalar products of a special type that we can represent in terms of determinants for chains of finite length $M$. We then calculate the thermodynamic limit of the form factors of the $\sigma^{z}$ operator in section 5. In particular, we show that if the rapidities of the particles and holes collapse on the Fermi surface then the form factors have a discrete structure. In section 6 we obtain analogous results for the form factors of the $\sigma^{ \pm}$operator:2 2 . We would like to stress that several methods and results used in the present article are borrowed from our previous work [1]. We conclude by discussing the possibility of applying our results to the calculation of the long-distance asymptotic behavior of the two-point functions at zero temperature. Several auxiliary Lemmas and proofs of technical character are presented in the two appendices.

\section{Space of states of the model}

Since our ultimate goal is to analyze the two-point correlation functions at zero temperature through (1.3), the form factors we consider here correspond to matrix elements of a local operator between the ground state and an excited eigenstate of the Hamiltonian (1.1). In this section we recall how such states can be described in the large $M$ limit.

In the algebraic Bethe Ansatz framework [11, 12, 13, the space of states of the model (in finite volume) is constructed by means of the Yang-Baxter algebra realized by the operator entries of the monodromy matrix. In the XXZ case, and more generally for the class of models associated with the six-vertex R-matrix, such monodromy matrices take the form

$$
T(\lambda)=\left(\begin{array}{ll}
A(\lambda) & B(\lambda) \\
C(\lambda) & D(\lambda)
\end{array}\right),
$$

where $A, B, C, D$ are quantum operators depending on some spectral parameter $\lambda$. It satisfies Yang-Baxter commutation relations given by the six-vertex R-matrix. In this framework, the eigenstates $|\psi\rangle$ of the Hamiltonian (1.1) coincide with the ones of the transfer matrix $\mathcal{T}(\lambda)=$ $\operatorname{tr} T(\lambda)=A(\lambda)+D(\lambda)$, and can be parameterized as $|\psi\rangle=\left|\psi\left(\lambda_{1}, \ldots, \lambda_{N}\right)\right\rangle, N=0,1, \ldots, M / 2$,

\footnotetext{
${ }^{2}$ Recall that $\sigma^{ \pm}=\frac{1}{2}\left(\sigma^{x} \pm i \sigma^{y}\right)$
} 
in terms of a set $\left\{\lambda_{j}\right\}_{j=1}^{N}$ of solutions to the logarithmic Bethe equations

$$
M p_{0}\left(\lambda_{j}\right)-\sum_{k=1}^{N} \vartheta\left(\lambda_{j}-\lambda_{k}\right)=2 \pi n_{j}, \quad j=1, \ldots, N .
$$

Here the functions $p_{0}(\lambda)$ and $\vartheta(\lambda)$ are the bare momentum and phase,

$$
p_{0}(\lambda)=i \log \left(\frac{\sinh \left(\frac{i \zeta}{2}+\lambda\right)}{\sinh \left(\frac{i \zeta}{2}-\lambda\right)}\right), \quad \vartheta(\lambda)=i \log \left(\frac{\sinh (i \zeta+\lambda)}{\sinh (i \zeta-\lambda)}\right),
$$

where $0<\zeta<\pi$ and $\cos \zeta=\Delta$. The numbers $n_{j},-M / 2<n_{j} \leq M / 2$, are integers (for $N$ odd) or half-integers (for $N$ even).

\subsection{Thermodynamic limit of the ground state}

The Bethe roots $\lambda_{j}, j=1, \ldots, N$, describing the ground state correspond to the solution of the system of logarithmic Bethe Ansatz equations (2.2) with the special choice $n_{j}=j-(N+1) / 2$, where $N$ is the number of down spins in the ground state $14,45,9,8$. Thus, for given $M$ and $h$, these parameters $\lambda_{j}$ are fixed quantities. In order to describe how they behave in the thermodynamic limit $(N, M \rightarrow \infty$ such that $N / M$ tends to some fixed average density $D)$, we introduce the ground state counting function,

$$
\widehat{\xi}(\omega)=\frac{1}{2 \pi} p_{0}(\omega)-\frac{1}{2 \pi M} \sum_{k=1}^{N} \vartheta\left(\omega-\lambda_{k}\right)+\frac{N+1}{2 M},
$$

which is build so that $\widehat{\xi}\left(\lambda_{j}\right)=j / M$ for $j=1, \ldots, N$. This function defines the discrete density of the minimal energy state in the $N$ sector by $\widehat{\rho}(\omega)=\widehat{\xi}^{\prime}(\omega)$. It is possible to argue that, in the thermodynamic limit, the parameters $\lambda_{j}$ fill densely a finite interval $[-q, q]$ of the real axis (the Fermi zone), and that the discrete density goes to a smooth function $\rho(\omega)$ that solves the following integral equation

$$
\rho(\lambda)+\frac{1}{2 \pi} \int_{-q}^{q} K(\lambda-\mu) \rho(\mu) d \mu=\frac{1}{2 \pi} p_{0}^{\prime}(\lambda), \quad \text { with } \quad K(\lambda)=\vartheta^{\prime}(\lambda) .
$$

The value of the endpoint $q$ of the Fermi zone is fixed by requiring that $\int_{-q}^{q} \rho(\lambda)=D$. Note that, since $\lim _{M \rightarrow \infty} \widehat{\xi}\left(\lambda_{1}\right)=0$, the thermodynamic limit $\xi(\lambda)$ of the counting function is the antiderivative of $\rho(\lambda)$ that vanishes at $-q$. Therefore $\xi(\lambda)$ can be expressed in terms of the dressed momentum $p(\lambda)$, the Fermi momentum being defined by $k_{F}=p(q)$

$$
p(\lambda)=2 \pi \int_{0}^{\lambda} \rho(\mu) d \mu, \quad \xi(\lambda)=[p(\lambda)+p(q)] / 2 \pi, \quad \pi D=p(q) \equiv k_{F} .
$$

\footnotetext{
${ }^{1}$ The number $N$ depends on the magnetization of the ground state, which is fixed by the overall magnetic field $h$. Hereafter, we call $N$ sector the subspace of the space of states having $N$ spins down.
} 
Several other properties of the ground state can be described through the solutions of other linear integral equations. We introduce two such functions that will play an important role in our analysis, namely the dressed charge $Z(\lambda)$ and the dressed phase $\phi(\lambda, \nu)$, which satisfy

$$
Z(\lambda)+\frac{1}{2 \pi} \int_{-q}^{q} K(\lambda-\mu) Z(\mu) d \mu=1
$$

and

$$
\phi(\lambda, \nu)+\frac{1}{2 \pi} \int_{-q}^{q} K(\lambda-\mu) \phi(\mu, \nu) d \mu=\frac{1}{2 \pi} \vartheta(\lambda-\nu) .
$$

In fact, these two functions are not independent. Using that $K(\lambda)=\vartheta^{\prime}(\lambda)$, one easily obtains that

$$
Z(\lambda)=1+\phi(\lambda, q)-\phi(\lambda,-q)
$$

Another non-trivial relationship between $Z$ and $\phi$ has been established in [46, 47]:

$$
1+\phi(q, q)-\phi(-q, q)=Z^{-1}(q)
$$

\subsection{Thermodynamic limit of the excited states}

To describe the other eigenstates of the transfer matrix $\mathcal{T}(\lambda)$, it is convenient for further purposes to consider instead the twisted transfer matrix $\mathcal{T}_{\kappa}(\lambda)=A(\lambda)+\kappa D(\lambda)$, where $\kappa$ is some complex (twist) parameter. Hereby the excited states of the Hamiltonian (1.1) are obtained as the $\kappa \rightarrow 1$ limit of the eigenstates of the twisted transfer matrix $\mathcal{T}_{\kappa}(\lambda)$ [27, 1].

In complete analogy with the standard algebraic Bethe Ansatz considerations, the eigenstates $\left|\psi_{\kappa}(\{\mu\})\right\rangle$ of $\mathcal{T}_{\kappa}(\lambda)$ can be parameterized by sets of solutions of the twisted Bethe equations

$$
M p_{0}\left(\mu_{\ell_{j}}\right)-\sum_{k=1}^{N_{\kappa}} \vartheta\left(\mu_{\ell_{j}}-\mu_{\ell_{k}}\right)=2 \pi\left(\ell_{j}-\frac{N_{\kappa}+1}{2}\right)+2 \pi \alpha, \quad j=1, \ldots, N_{\kappa} .
$$

Such states $\left|\psi_{\kappa}(\{\mu\})\right\rangle$ depend on $N_{\kappa}$ parameters $\left\{\mu_{\ell_{k}}\right\}_{k=1}^{N_{\kappa}}$, where $N_{\kappa}$ is the number of spins down and we have set $\kappa=e^{2 \pi i \alpha}$. In this article we consider $\alpha$ to be a real number. This restriction $\Im(\alpha)=0$ is not crucial, but convenient, since then $\left\langle\psi_{\kappa}(\{\mu\})\left|=(-1)^{N_{\kappa}}\right| \psi_{\kappa}(\{\mu\})\right\rangle^{\dagger}$.

Remark 2.1. One could of course from the very beginning set $\alpha=0$ and deal directly with the standard excited states of the XXZ Hamiltonian (1.1). However, we chose to introduce this extra parameter and keep it throughout our computations since, as we will show later on, an important class of form factors can be obtained by a mere shift of $\alpha$ by some integer value.

In the following, we will consider two cases of interest: $N_{\kappa}=N$ for form factors of $\sigma^{z}$, and $N_{\kappa}=N+1$ for the $\sigma^{+}$form factors]. It follows from [8] that the set of real roots of the twisted Bethe equations is completely defined by the choice of the set of integers $\ell_{j}$ in the r.h.s. of (2.11). Therefore, we have labeled the roots of (2.11) by subscripts $\ell_{j}$.

\footnotetext{
${ }^{1}$ We do not consider the case $N_{\kappa}=N-1$ corresponding to form factors of $\sigma^{-}$, since the last ones can be obtained from the form factors of $\sigma^{+}$.
} 
Definition 2.1. The state parameterized by the real solutions to (2.11) with $\ell_{j}=j, j=$ $1, \ldots, N_{\kappa}$, is called $\alpha$-twisted ground state in the $N_{\kappa}$ sector.

In the thermodynamic limit, excitations corresponding to real set of solutions to the twisted Bethe equations above this $\alpha$-twisted ground state can be described in terms of particles and holes. Note that the states associated to complex roots of the Bethe equations (like string solutions) are not considered in the present article. They correspond in particular to form factors of bound states [5, 6, 7, 48, that will be dealt with in a separate publication. All this means that one deals with solutions to the twisted Bethe equations where most of the $\ell_{j}$ coincide with their value for the ground state: $\ell_{j}=j$ except for $n$ integers for which $\ell_{j} \neq j$, with $n$ remaining finite in the $N_{\kappa} \rightarrow \infty$ limit. To be more precise, we fix $2 n$ distinct integers $h_{1}, \ldots, h_{n}$ and $p_{1}, \ldots, p_{n}$ such that $h_{k} \in\left\{1, \ldots, N_{\kappa}\right\}$ and $p_{k} \notin\left\{1, \ldots, N_{\kappa}\right\}$. The integers $h_{k}$ represent "holes" in respect to the distributions of integers for the ground state in the $N_{\kappa}$ sector, whereas the integers $p_{k}$ represent "particles". In other words, the excited state is described by a set of integers $\left\{\ell_{j}\right\}_{j=1}^{N_{\kappa}}$ such that $\ell_{j}=j$ for $j \neq h_{1}, \ldots, h_{n}$, and $\ell_{h_{k}}=p_{k}$.

The terminology of particles and holes takes a clearer interpretation in terms of the counting function $\widehat{\xi}_{\kappa}$ associated with the $N_{\kappa}$-excited state:

$$
\widehat{\xi}_{\kappa}(\omega)=\frac{1}{2 \pi} p_{0}(\omega)-\frac{1}{2 \pi M} \sum_{k=1}^{N_{\kappa}} \vartheta\left(\omega-\mu_{\ell_{k}}\right)+\frac{N_{\kappa}+1}{2 M}-\frac{\alpha}{M} .
$$

One can argue that the counting function is monotonously increasing on the real axis. Thus it is possible to define unambiguously a set of real parameters $\mu_{j}$ as the unique solutions to $\widehat{\xi}_{\kappa}\left(\mu_{j}\right)=j / M$. One can identify, among this set of parameters, the $N_{\kappa}$ solutions of the twisted Bethe equations with integers $\ell_{j}$ as being $\mu_{\ell_{j}}$ as it should be. In this picture, an excited state no-longer corresponds to the set of $N_{\kappa}$ consecutive solutions $\mu_{j}$ to the equation $\widehat{\xi}_{\kappa}\left(\mu_{j}\right)=j / M$, but is rather obtained by removing the solutions $\widehat{\xi}_{\kappa}\left(\mu_{h_{a}}\right)=h_{a} / M$ and replacing them by the solutions $\widehat{\xi}_{\kappa}\left(\mu_{p_{a}}\right)=p_{a} / M$. It is in this respect that $\mu_{h_{a}}$ stands for the rapidities of the holes and that $\mu_{p_{a}}$ stands for those of the particles. From now on, we agree upon $\mu_{j}$ being the solution to $\widehat{\xi}_{\kappa}\left(\mu_{j}\right)=j / M$ with $\widehat{\xi}_{\kappa}$ being given by (2.12).

Similarly as for the ground state in the $N$ sector, one can also define the discrete density of the excited state by $\widehat{\rho}_{\kappa}(\omega)=\widehat{\xi}_{\kappa}^{\prime}(\omega)$. It is easy to see that the thermodynamic limits of this new counting function and its associated density coincide with the limits defined above in (2.5), (2.6).

An excited state is most conveniently characterized in terms of the shift function $\widehat{F}(\omega)=$ $\widehat{F}\left(\omega\left|\left\{\mu_{p}\right\}\right|\left\{\mu_{h}\right\}\right)$ defined by

$$
\widehat{F}(\omega)=M\left(\widehat{\xi}(\omega)-\widehat{\xi}_{\kappa}(\omega)\right)
$$

The shift function describes the spacing between the root $\lambda_{j}$ for the ground state in the $N$ sector and the parameters $\mu_{j}$ defined by $\widehat{\xi}_{\kappa}\left(\mu_{j}\right)=j / M$ :

$$
\mu_{j}-\lambda_{j}=\frac{F\left(\lambda_{j}\right)}{\rho\left(\lambda_{j}\right) M}+O\left(M^{-2}\right)
$$

where $F(\lambda)$ is the thermodynamic limit of the shift function. Recall that we consider the excited states in the $N_{\kappa}$ sector with $N_{\kappa}=N$ (for form factors of $\sigma^{z}$ ) and $N_{\kappa}=N+1$ (for 
form factors of $\left.\sigma^{+}\right)$. Respectively we should distinguish between two shift functions $F^{(z)}(\lambda)$ and $F^{(+)}(\lambda)$ corresponding to these two cases. Generically the shift function $F(\lambda)$ satisfies the integral equation

$$
F(\lambda)+\int_{-q}^{q} K(\lambda-\mu) F(\mu) \frac{d \mu}{2 \pi}=\alpha+\frac{\delta N}{2}\left[1-\frac{\vartheta(\lambda-q)}{\pi}\right]+\frac{1}{2 \pi} \sum_{k=1}^{n}\left[\vartheta\left(\lambda-\mu_{p_{k}}\right)-\vartheta\left(\lambda-\mu_{h_{k}}\right)\right] .
$$

where $\delta N=N-N_{\kappa}$. Using (2.7) and (2.8) we conclude that

$$
F^{(z)}(\lambda)=\alpha Z(\lambda)+\sum_{k=1}^{n} \phi\left(\lambda, \mu_{p_{k}}\right)-\sum_{k=1}^{n} \phi\left(\lambda, \mu_{h_{k}}\right) .
$$

and

$$
F^{(+)}(\lambda)=\left(\alpha-\frac{1}{2}\right) Z(\lambda)+\sum_{k=1}^{n} \phi\left(\lambda, \mu_{p_{k}}\right)-\sum_{k=1}^{n} \phi\left(\lambda, \mu_{h_{k}}\right)+\phi(\lambda, q)
$$

\section{The main results}

We now formulate a precise statement of the results obtained in this article concerning the thermodynamic behavior of form factors. In fact, for later use, we will give this thermodynamic behavior directly for the products of form factors appearing in the spectral expansion of the correlation function (1.3). However, it will be made clear that our method allows also to obtain the behavior of each individual form factor. The proof of the results we present below will be given in the next sections.

We consider the form factors (1.2) of the local spin operators $\sigma_{m}^{s}, s=+,-, z$, between the ground state $\left|\psi_{g}\right\rangle$ and some (different) excited state $\left|\psi^{\prime}\right\rangle$ of the Hamiltonian 1 . Here and in the following, we set $\left|\psi_{g}\right\rangle \equiv|\psi(\{\lambda\})\rangle,\left\{\lambda_{1}, \ldots, \lambda_{N}\right\}$ being the solution of the Bethe equations (2.2) describing the ground state of the Hamiltonian, and $\left|\psi^{\prime}\right\rangle=\lim _{\kappa \rightarrow 1}\left|\psi_{\kappa}(\{\mu\})\right\rangle$ for $\left\{\mu_{\ell_{1}}, \ldots, \mu_{\ell_{N_{\kappa}}}\right\}$ being a solution of the $\kappa$-twisted Bethe equations (2.11) with $n$ particles and $n$ holes. We will be specially interested in the limiting case where, in the thermodynamic limit, all rapidities $\left\{\mu_{p_{j}}\right\}_{j=1}^{n}$ and $\left\{\mu_{h_{j}}\right\}_{j=1}^{n}$ of particles and holes condensate on the Fermi boundaries. Such excited states have a zero excitation energy in the thermodynamic limit; we expect that they will produce the main contribution to the asymptotic behavior of correlation functions (this fact is already apparent in the predictions based on the Luttinger liquid theory and Conformal Field Theory for the long-distance asymptotic behavior of two-point functions [37, 38, 39, 40, 41, 42, 43, 44]). We will also consider the opposite limiting case, namely when all particle/hole rapidities remain at finite distance from the Fermi boundaries.

In the thermodynamic limit, up to uniformly $M^{-1} \cdot \log M$ corrections, the products of such form factors behave as

$$
\begin{aligned}
& \left.\mathcal{F}_{\psi_{g} \psi^{\prime}}^{(z)}\left(m^{\prime}\right) \cdot \mathcal{F}_{\psi^{\prime} \psi_{g}}^{(z)}(m) \sim \delta_{N, N_{\kappa}} M^{-\theta_{z z}} e^{i \mathcal{P}_{e x}\left(m-m^{\prime}\right)} \partial_{\alpha}^{2} \mathcal{S}_{z z} \mathcal{D}_{z z}\right|_{\alpha=0} \\
& \left.\mathcal{F}_{\psi_{g} \psi^{\prime}}^{(+)}\left(m^{\prime}\right) \cdot \mathcal{F}_{\psi^{\prime} \psi_{g}}^{(-)}(m) \sim \delta_{N+1, N_{\kappa}} M^{-\theta_{+-}}(-1)^{m-m^{\prime}} e^{i \mathcal{P}_{e x}\left(m-m^{\prime}\right)} \mathcal{S}_{+-} \mathcal{D}_{+-}\right|_{\alpha=0}
\end{aligned}
$$

\footnotetext{
${ }^{1}$ The case when $\left|\psi^{\prime}\right\rangle=\left|\psi_{g}\right\rangle$ is trivial: $\mathcal{F}_{\psi_{g} \psi_{g}}^{( \pm)}(m)=0, \mathcal{F}_{\psi_{g} \psi_{g}}^{(z)}(m)=2 D-1$.
} 
Let us explain these formulas. These products decrease as some negative power of the size $M$ of the system, the exponents $\theta_{z z}, \theta_{+-}$being specified below. Due to the translation invariance of the model, all the dependence on the lattice spacing $m-m^{\prime}$ is absorbed into a phase factor, with

$$
\mathcal{P}_{\text {ex }}=\lim _{M, N \rightarrow+\infty}\left\{\sum_{j=1}^{N_{\kappa}} p_{0}\left(\mu_{\ell_{j}}\right)-\sum_{j=1}^{N} p_{0}\left(\lambda_{j}\right)\right\}=2 \pi \alpha D+\sum_{j=1}^{n}\left[p\left(\mu_{p_{j}}\right)-p\left(\mu_{h_{j}}\right)\right]
$$

being the momentum of the excited state relative to the ground state. We have split the constant in front of the $M$ and $\left(m-m^{\prime}\right)$ dependence in two parts, $\mathcal{S}_{s s^{\prime}}$ and $\mathcal{D}_{s s^{\prime}}$, that we call smooth and discrete parts respectively. The reason for this denomination is that the smooth part $\mathcal{S}_{s s^{\prime}}$ depends continuously on the rapidities $\mu_{p_{j}}$ and $\mu_{h_{j}}$ of the particles and holes, whereas the discrete part $\mathcal{D}_{s s^{\prime}}$ also depends on the set of integers appearing in the logarithmic Bethe Ansatz equations (2.11) for the excited state. Such a discrete structure can be fairly well approximated by a smooth function of the rapidities $\mu_{p_{j}}$ and $\mu_{h_{j}}$ as long as they are located at a finite distance from the Fermi boundaries. However, as soon as the rapidities of the particles or holes approach the Fermi surface, the discrete structure of the form factors can no longer be neglected: a microscopic (of order $1 / M$ ) deviation of a particle (or hole) rapidity leads to a macroscopic change in $\mathcal{D}_{s s^{\prime}}$.

\subsection{Smooth parts}

The smooth parts $\mathcal{S}_{z z}$ and $\mathcal{S}_{+-}$are decomposed into

$$
\mathcal{S}_{z z}=\frac{2}{\pi^{2}} \sin ^{2}\left(\frac{\mathcal{P}_{e x}}{2}\right) \cdot \mathcal{A}_{n}^{(z)} \cdot e^{C_{n}^{(z)}}, \quad \mathcal{S}_{+-}=\mathcal{A}_{n}^{(+)} \cdot e^{C_{n}^{(+)}} .
$$

Here $C_{n}^{(z)}, \mathcal{A}_{n}^{(z)}$ are functionals acting on the shift function $F^{(z)}(\lambda)$ given in (2.16), while $C_{n}^{(+)}$, $\mathcal{A}_{n}^{(+)}$are functionals acting on the shift function $F^{(+)}(\lambda)$ given in (2.17). This action is such that the result depends smoothly on the particle/hole rapidities $\left\{\mu_{p}\right\}$ and $\left\{\mu_{h}\right\}$ of the state $\left|\psi_{\kappa}(\{\mu\})\right\rangle$.

Before giving explicit expressions for the quantities in (3.3) we introduce the $i \pi$-periodic Cauchy transform of the shift functions $F^{(z /+)}(\lambda)$ on $[-q, q]$,

$$
\mathbf{F}^{(z /+)}(w)=\frac{1}{2 \pi i} \int_{-q}^{q} F^{(z /+)}(\lambda) \operatorname{coth}(\lambda-w) d \lambda .
$$

Then the coefficient $C_{n}^{(z)}=C_{n}^{(z)}\left(\left\{\mu_{p}\right\},\left\{\mu_{h}\right\}\right)$ has the form

$$
\begin{aligned}
C_{n}^{(z)}\left[F^{(z)}\right]=C_{0}\left[F^{(z)}\right]+2 \pi i \sum_{j=1}^{n}\left(\mathbf{F}^{(z)}\left(\mu_{h_{j}}-i \zeta\right)+\mathbf{F}^{(z)}\left(\mu_{h_{j}}+i \zeta\right)-\mathbf{F}^{(z)}\left(\mu_{p_{j}}-i \zeta\right)-\mathbf{F}^{(z)}\left(\mu_{p_{j}}+i \zeta\right)\right) \\
\quad+\sum_{j, k=1}^{n} \log \frac{\sinh \left(\mu_{h_{j}}-\mu_{p_{k}}-i \zeta\right) \sinh \left(\mu_{p_{k}}-\mu_{h_{j}}-i \zeta\right)}{\sinh \left(\mu_{p_{j}}-\mu_{p_{k}}-i \zeta\right) \sinh \left(\mu_{h_{j}}-\mu_{h_{k}}-i \zeta\right)}, \quad \text { (3.5) }
\end{aligned}
$$


where the functional $C_{0}[F]$ reads

$$
C_{0}[F]=-\int_{-q}^{q} \frac{F(\lambda) F(\mu)}{\sinh ^{2}(\lambda-\mu-i \zeta)} d \lambda d \mu .
$$

The coefficient $C_{n}^{(+)}=C_{n}^{(+)}\left(\left\{\mu_{p}\right\},\left\{\mu_{h}\right\}\right)$ has similar representation, but it contains several additional terms

$$
\begin{aligned}
C_{n}^{(+)}\left[F^{(+)}\right]=C_{n}^{(z)}\left[F^{(+)}\right]-2 \pi i\left(\mathbf{F}^{(+)}(q\right. & \left.+i \zeta)+\mathbf{F}^{(+)}(q-i \zeta)\right) \\
& +\sum_{j=1}^{n} \log \frac{\sinh \left(\mu_{h_{j}}-q-i \zeta\right) \sinh \left(\mu_{h_{j}}-q+i \zeta\right)}{\sinh \left(\mu_{p_{j}}-q-i \zeta\right) \sinh \left(\mu_{p_{j}}-q+i \zeta\right)}
\end{aligned}
$$

The functional $\mathcal{A}_{n}^{(z)}=\mathcal{A}_{n}^{(z)}\left(\left\{\mu_{p}\right\},\left\{\mu_{h}\right\}\right)$ has the form

$$
\begin{aligned}
\mathcal{A}_{n}^{(z)}\left[F^{(z)}\right]=\left(\frac{\sin \pi \alpha}{\sin \pi F^{(z)}(-q)}\right)^{2}\left|e^{-2 \pi i \mathbf{F}^{(z)}(-q+i \zeta)} \prod_{k=1}^{n} \frac{\sinh \left(q+\mu_{h_{k}}+i \zeta\right)}{\sinh \left(q+\mu_{p_{k}}+i \zeta\right)}\right|^{2} & \times\left|\frac{\operatorname{det}\left[I+\frac{1}{2 \pi i} U^{(z)}\left(w, w^{\prime}\right)\right]}{\operatorname{det}\left[I+\frac{1}{2 \pi} K\right]}\right|^{2}
\end{aligned}
$$

The equation (3.8) contains a ratio of Fredholm determinants. The integral operator $I+\frac{1}{2 \pi} K$ acts on the interval $[-q, q]$, whereas the integral operators $I+\frac{1}{2 \pi i} U^{(z)}$ acts on a counterclockwise oriented contour $\Gamma_{q}$ surrounding $[-q, q]$. This contour is such that it contains all the ground state roots and no other singularity of the kernel. The integral kernel is defined by

$$
U^{(z)}\left(w, w^{\prime}\right)=-\Phi(w) e^{2 \pi i\left(\mathbf{F}^{(z)}(w)-\mathbf{F}^{(z)}(w+i \zeta)\right)} \frac{K_{\kappa}\left(w-w^{\prime}\right)-K_{\kappa}\left(-q-w^{\prime}\right)}{1-e^{2 \pi i F^{(z)}(w)}},
$$

with

$$
K_{\kappa}(w)=\operatorname{coth}(w+i \zeta)-\kappa \operatorname{coth}(w-i \zeta), \quad \Phi(w)=\prod_{k=1}^{n} \frac{\sinh \left(w-\mu_{p_{k}}\right) \sinh \left(w-\mu_{h_{k}}+i \zeta\right)}{\sinh \left(w-\mu_{h_{k}}\right) \sinh \left(w-\mu_{p_{k}}+i \zeta\right)}
$$

The coefficient $\mathcal{A}_{n}^{(+)}=\mathcal{A}_{n}^{(+)}\left(\left\{\mu_{p}\right\},\left\{\mu_{h}\right\}\right)$ also is proportional to a ratio of Fredholm determinants

$$
\mathcal{A}_{n}^{(+)}\left[F^{(+)}\right]=\frac{\sin \zeta}{2 \pi \kappa}\left|\frac{e^{-2 \pi i \mathbf{F}^{(+)}\left(\frac{i \zeta}{2}\right)}}{\sinh \left(q-\frac{i \zeta}{2}\right)} \prod_{k=1}^{n} \frac{\sinh \left(\mu_{h_{k}}-\frac{i \zeta}{2}\right)}{\sinh \left(\mu_{p_{k}}-\frac{i \zeta}{2}\right)} \cdot \frac{\operatorname{det}\left[I+\frac{1}{2 \pi i} U^{(+)}\left(w, w^{\prime}\right)\right]}{\operatorname{det}\left[I+\frac{1}{2 \pi} K\right]}\right|^{2} .
$$

Similarly to the integral operator defined in (3.9), the operator $I+\frac{1}{2 \pi i} U^{(+)}$acts on the contour $\Gamma_{q}$ surrounding $[-q, q]$, with a kernel

$$
U^{(+)}[F]\left(w, w^{\prime}\right)=\Phi(w) \frac{\sinh (w-q)}{\sinh (w-q+i \zeta)} e^{2 \pi i\left(\mathbf{F}^{(+)}(w)-\mathbf{F}^{(+)}(w+i \zeta)\right)} \frac{\mathcal{K}_{\kappa}\left(w, w^{\prime}\right)}{1-e^{2 \pi i F^{(+)}(w)}},
$$

where

$$
\mathcal{K}_{\kappa}\left(w, w^{\prime}\right)=\frac{\sinh \left(w^{\prime}+\frac{3 i \zeta}{2}\right)}{\sinh \left(w^{\prime}-\frac{i \zeta}{2}\right) \sinh \left(w-w^{\prime}-i \zeta\right)}-\frac{\kappa \sinh \left(w^{\prime}-\frac{3 i \zeta}{2}\right)}{\sinh \left(w^{\prime}+\frac{i \zeta}{2}\right) \sinh \left(w-w^{\prime}+i \zeta\right)},
$$

and $\Phi(w)$ is given by (3.10). 


\subsection{Discrete parts and exponents}

The values of the discrete parts $\mathcal{D}_{z z}$ and $\mathcal{D}_{+-}$, as well as of the exponents $\theta_{z z}$ and $\theta_{+-}$, depend on whether particles and holes are on the Fermi surface or separated from it. We specify their expressions in the two particular cases we mentioned above: when all particle/hole rapidities remain at finite distance from the Fermi boundaries, or when all particle/hole rapidities collapse on the Fermi boundaries. Other intermediate configurations can be obtained along similar techniques.

\subsubsection{Particles and holes away from the Fermi boundaries}

In the first case, the exponents in (3.1) giving the algebraic decay of the form factor with the system size read:

$$
\begin{aligned}
& \theta_{z z}=2 n+\left(F_{+}^{(z)}\right)^{2}+\left(F_{-}^{(z)}\right)^{2}, \\
& \theta_{+-}=2 n+\left(F_{+}^{(+)}+1\right)^{2}+\left(F_{-}^{(+)}\right)^{2}
\end{aligned}
$$

where $F_{ \pm}^{(z /+)}=F^{(z /+)}( \pm q)$ are given in terms of the shift functions defined in (2.16) and (2.17). The discrete part $\mathcal{D}_{z z}$ is given by

$$
\begin{aligned}
\mathcal{D}_{z z}\left(\left\{\mu_{p}\right\},\left\{\mu_{h}\right\}\right)=[\operatorname{det} & \left.\frac{1}{\sinh \left(\mu_{p_{j}}-\mu_{h_{k}}\right)}\right]^{2} \cdot \mathcal{D}^{(z)}\left[F^{(z)}\right] \prod_{k=1}^{n} \frac{\sin ^{2}\left(\pi F^{(z)}\left(\mu_{h_{k}}\right)\right)}{\pi^{2} \rho\left(\mu_{h_{k}}\right) \rho\left(\mu_{p_{k}}\right)} \\
& \times \prod_{k=1}^{n} \exp \left\{2 \oint_{-q}^{q} F^{(z)}(\lambda)\left[\operatorname{coth}\left(\lambda-\mu_{p_{k}}\right)-\operatorname{coth}\left(\lambda-\mu_{h_{k}}\right)\right] d \lambda\right\} .
\end{aligned}
$$

Here a functional $\mathcal{D}^{(z)}[F]$ has the following form:

$$
\mathcal{D}^{(z)}[F]=\frac{G^{2}\left(1-F_{-}\right) G^{2}\left(1+F_{+}\right)(2 \pi)^{F_{-}-F_{+}}}{[\rho(q) \sinh (2 q)]^{\left(F_{-}\right)^{2}+\left(F_{+}\right)^{2}}} e^{C_{1}[F]} .
$$

where $G(z)$ is the Barnes function satisfying $G(z+1)=\Gamma(z) G(z)$, and we have set

$$
\begin{aligned}
C_{1}[F]=\int_{-q}^{q} \frac{F^{\prime}(\lambda) F(\mu)-F(\lambda) F^{\prime}(\mu)}{2 \tanh (\lambda-\mu)} d \lambda d \mu & \\
& +F_{+} \int_{-q}^{q} \frac{F_{+}-F(\lambda)}{\tanh (q-\lambda)} d \lambda+F_{-} \int_{-q}^{q} \frac{F_{-}-F(\lambda)}{\tanh (q+\lambda)} d \lambda .
\end{aligned}
$$

The discrete part $\mathcal{D}_{+-}$has the form

$$
\begin{aligned}
& \mathcal{D}_{+-}\left(\left\{\mu_{p}\right\},\left\{\mu_{h}\right\}\right)=\left[\operatorname{det} \frac{1}{\sinh \left(\mu_{p_{j}}-\mu_{h_{k}}\right)}\right]^{2} \cdot \mathcal{D}^{(+)}\left[F^{(+)}\right] \prod_{k=1}^{n} \frac{\sin ^{2}\left(\pi F^{(+)}\left(\mu_{h_{k}}\right)\right)}{\pi^{2} \rho\left(\mu_{h_{k}}\right) \rho\left(\mu_{p_{k}}\right)} \\
& \times \prod_{k=1}^{n}\left[\frac{\sinh \left(\mu_{p_{k}}-q\right)}{\sinh \left(\mu_{h_{k}}-q\right)}\right]^{2} \exp \left\{2 \oint_{-q}^{q} F^{(+)}(\lambda)\left[\operatorname{coth}\left(\lambda-\mu_{p_{k}}\right)-\operatorname{coth}\left(\lambda-\mu_{h_{k}}\right)\right] d \lambda\right\},
\end{aligned}
$$


where the functional $\mathcal{D}^{(+)}[F]$ reads

$$
\mathcal{D}^{(+)}[F]=\mathcal{D}^{(z)}[F] \cdot \frac{\sinh (2 q) \Gamma^{2}\left(1+F_{+}\right)}{[\rho(q) \sinh (2 q)]^{1+2 F_{+}}} \exp \left\{2 \int_{-q}^{q} \frac{F_{+}-F(\lambda)}{\tanh (q-\lambda)} d \lambda\right\} .
$$

\subsubsection{Particles and holes on the Fermi boundaries}

Definition 3.1. Let an excited state contain $n$ particles and $n$ holes. It is called critical excited state, if all the rapidities $\mu_{p}, \mu_{h}= \pm q$ in the thermodynamic limit. We also say that this excited state belongs to the $\mathbf{P}_{r}$ class if it contains $n_{p}^{ \pm}$particles, resp. $n_{h}^{ \pm}$holes, with rapidities equal to $\pm q$ such that

$$
n_{p}^{+}-n_{h}^{+}=n_{h}^{-}-n_{p}^{-}=r, \quad r \in \mathbb{Z} .
$$

The corresponding form factors are called critical form factors of the $\mathbf{P}_{r}$ class.

The rapidities of the particles and holes in an excited state belonging to the $\mathbf{P}_{r}$ class are all located in a close neighborhood of $\pm q$. As a consequence, it is useful to re-parameterize the integers describing the position of particles and holes according to

$$
\begin{array}{lll}
p_{j}=p_{j}^{+}+N_{\kappa}, & \text { if } \quad \mu_{p_{j}}=q, \\
p_{j}=1-p_{j}^{-}, & \text {if } \quad \mu_{p_{j}}=-q, \\
h_{j}=N_{\kappa}+1-h_{j}^{+}, & \text {if } \quad \mu_{h_{j}}=q, \\
h_{j}=h_{j}^{-}, & \text {if } \quad \mu_{h_{j}}=-q .
\end{array}
$$

All the integers $\left\{p^{ \pm}\right\}$and $\left\{h^{ \pm}\right\}$introduced above are positive and vary in a range such that

$$
\lim _{N \rightarrow \infty} \frac{\sum p_{j}^{ \pm}}{N}=\lim _{N \rightarrow \infty} \frac{\sum h_{j}^{ \pm}}{N}=0
$$

which means that $\mu_{p}$ and $\mu_{h}$ indeed collapse to the Fermi boundary in the thermodynamic limit.

For $\sigma^{z}$ and $\sigma^{ \pm}$form factors belonging to the $\mathbf{P}_{r}$ class, the discrete parts are given by

$$
\begin{array}{r}
\mathcal{D}_{z z}=\mathcal{D}^{(z)}\left[F_{r}^{(z)}\right] \frac{G^{2}\left(1+F_{+}^{(z)}\right) G^{2}\left(1-F_{-}^{(z)}\right)}{G^{2}\left(1+F_{r,+}^{(z)}\right) G^{2}\left(1-F_{r,-}^{(z)}\right)}\left(\frac{\sin \left(\pi F_{r,+}^{(z)}\right)}{\pi}\right)^{2 n_{h}^{+}}\left(\frac{\sin \left(\pi F_{r,-}^{(z)}\right)}{\pi}\right)^{2 n_{h}^{-}} \\
\times R_{n_{p}^{+}, n_{h}^{+}}\left(\left\{p^{+}\right\},\left\{h^{+}\right\} \mid F_{+}^{(z)}\right) R_{n_{p}^{-}, n_{h}^{-}}\left(\left\{p^{-}\right\},\left\{h^{-}\right\} \mid-F_{-}^{(z)}\right),
\end{array}
$$

and

$$
\begin{aligned}
\mathcal{D}_{+-}=\mathcal{D}^{(+)}\left[F_{r}^{(+)}\right] \frac{G^{2}\left(2+F_{+}^{(+)}\right) G^{2}\left(1-F_{-}^{(+)}\right)}{G^{2}\left(2+F_{r,+}^{(+)}\right) G^{2}\left(1-F_{r,-}^{(+)}\right)}\left(\frac{\sin \left(\pi F_{r,+}^{(+)}\right)}{\pi}\right)^{2 n_{h}^{+}}\left(\frac{\sin \left(\pi F_{r,-}^{(+)}\right)}{\pi}\right)^{2 n_{h}^{-}} \\
\times R_{n_{p}^{+}, n_{h}^{+}}\left(\left\{p^{+}\right\},\left\{h^{+}\right\} \mid 1+F_{+}^{(+)}\right) R_{n_{p}^{-}, n_{h}^{-}}\left(\left\{p^{-}\right\},\left\{h^{-}\right\} \mid-F_{-}^{(+)}\right) .
\end{aligned}
$$

Here we agree upon $F_{r}^{(z /+)}(\lambda)=F^{(z /+)}(\lambda)+r$ and $F_{r, \pm}^{(z /+)}=F_{r}^{(z /+)}( \pm q)$. The functionals $\mathcal{D}^{(z)}$ and $\mathcal{D}^{(+)}$are given respectively in (3.16) and (3.19). The coefficient $R_{n, m}(\{p\},\{h\} \mid F)$ is 
defined as

$$
R_{n, m}(\{p\},\{h\} \mid F)=\frac{\prod_{j>k}^{n}\left(p_{j}-p_{k}\right)^{2} \prod_{j>k}^{m}\left(h_{j}-h_{k}\right)^{2}}{\prod_{j=1}^{n} \prod_{k=1}^{m}\left(p_{j}+h_{k}-1\right)^{2}} \Gamma^{2}\left(\begin{array}{cl}
\left\{p_{k}+F\right\} & ,\left\{h_{k}-F\right\} \\
\left\{p_{k}\right\} & ,\left\{h_{k}\right\}
\end{array}\right),
$$

where we have used the standard hypergeometric type notation for ratios of $\Gamma$ functions:

$$
\Gamma\left(\begin{array}{c}
a_{1}, \ldots, a_{\ell} \\
b_{1}, \ldots, b_{j}
\end{array}\right)=\prod_{k=1}^{\ell} \Gamma\left(a_{k}\right) \cdot \prod_{k=1}^{j} \Gamma\left(b_{k}\right)^{-1} .
$$

The exponents $\theta$ in this case are

$$
\begin{aligned}
& \theta_{z z}(r)=\left(F_{r,+}^{(z)}\right)^{2}+\left(F_{r,-}^{(z)}\right)^{2}, \\
& \theta_{+-}(r)=\left(1+F_{r,+}^{(+)}\right)^{2}+\left(F_{r,-}^{(+)}\right)^{2} .
\end{aligned}
$$

Note that the shift functions $F^{(z /+)}(\lambda)$ enter the exponents (3.27) only in the combination $F_{r}^{(z /+)}(\lambda)=F^{(z /+)}(\lambda)+r$. It is easy to see that $F_{r}^{(z /+)}(\lambda)$ are nothing else but the shift functions of the $(\alpha+r)$-twisted ground states (see Definition 2.1) in the $N$ and $N+1$ sectors respectively.

Remark 3.1. One can write down the results of this section in a more symmetric form. For this we introduce two constants $f_{\mp}$ describing the shift of the utmost parameters $\mu_{1}$ and $\mu_{N_{\kappa}}$ of the $(\alpha+r)$-twisted ground state with respect to the utmost parameters $\lambda_{1}$ and $\lambda_{N}$ of the ground state:

$$
\begin{aligned}
& f_{-}=\lim _{N, M \rightarrow \infty} M \widehat{\rho}\left(\lambda_{1}\right)\left(\mu_{1}-\lambda_{1}\right), \\
& f_{+}=\lim _{N, M \rightarrow \infty} M \widehat{\rho}\left(\lambda_{N}\right)\left(\mu_{N_{\kappa}}-\lambda_{N}\right) .
\end{aligned}
$$

Then it is easy to see that $f_{ \pm}=F_{r, \pm}^{(z)}$ for the $\sigma^{z}$ form factors. However for the $\sigma^{+}$form factors (where $N_{\kappa}=N+1$ ) one has $f_{-}=F_{r,-}^{(+)}$, but $f_{+}=1+F_{r,+}^{(+)}$. Then in both cases the exponents (3.27) can be written as $\theta=f_{+}^{2}+f_{-}^{2}$.

It is crucial to compare the results (3.27) with the critical exponents appearing in the asymptotic behavior of the two-point correlation functions predicted in [37, 38, 39, 40, 41, 42, 43, 44, 49, 50, 36.

In the case of $\theta_{z z}(r)$, it follows from (2.9), (2.16) that $F_{r}^{(z)}(\lambda)=(\alpha+r) Z(\lambda)$. Therefore, for $\alpha=0$, we obtain that $\theta_{z z}(r)=2 r^{2} Z^{2}(q),|r|=1,2, \ldots$ These numbers coincide with the critical exponents appearing in the asymptotic behavior of the $\left\langle\sigma_{1}^{z} \sigma_{m+1}^{z}\right\rangle$ correlation function and are associated to the oscillating term with momentum $2 r k_{F}$.

The exponent $\theta_{+-}(r)$ has similar properties. In this case, $F_{r}^{(+)}(\lambda)=(\alpha+r-1 / 2) Z(\lambda)+$ $\phi(\lambda, q)$. It follows from equations (2.9) and (2.10) that

$$
\begin{aligned}
& 1+\phi(q, q)=\frac{Z(q)+Z^{-1}(q)}{2}, \\
& \phi(-q, q)=\frac{Z(q)-Z^{-1}(q)}{2} .
\end{aligned}
$$


From this we find

$$
\begin{aligned}
& F_{r,+}+1=(\alpha+r) Z(q)+\frac{Z^{-1}(q)}{2}, \\
& F_{r,-}=(\alpha+r) Z(q)-\frac{Z^{-1}(q)}{2} .
\end{aligned}
$$

Thus, at $\alpha=0$ we have $\theta_{+-}(r)=Z^{-2}(q) / 2+2 r^{2} Z^{2}(q),|r|=0,1, \ldots$ Again, these numbers coincide with the critical exponents appearing in the asymptotic behavior of the $\left\langle\sigma_{1}^{+} \sigma_{m+1}^{-}\right\rangle$ correlation function and associated to the oscillating term with momentum $2 r k_{F}$. Thus, we see that the behavior of critical form factors with respect to the size of the system coincides with the asymptotic behavior of two-point correlation functions with respect to the lattice distance.

\section{Form factors and scalar products}

The solution of the quantum inverse scattering problem enables us to express the local spin operators in terms of the entries of the monodromy matrix [10, 23]:

$$
\sigma_{m}^{s}=\mathcal{T}^{m-1}(-i \zeta / 2) \cdot \operatorname{tr}\left(T(-i \zeta / 2) \sigma^{s}\right) \cdot \mathcal{T}^{-m}(-i \zeta / 2) .
$$

In the l.h.s. of this expression, the symbol $\sigma_{m}^{s}(s= \pm, z)$ denotes the corresponding local spin operator at site $m$, whereas the symbol $\sigma^{s}$ appearing in the r.h.s. should be understood as a $2 \times 2$ Pauli matrix multiplying the $2 \times 2$ monodromy matrix (2.1) .

Using (4.1), one can reduce the computation of the form factors of local spin operators in the finite XXZ chain to the one of the scalar products [10]. We have

$$
\begin{aligned}
& \mathcal{F}_{\psi^{\prime} \psi_{g}}^{(z)}(m)=-e^{i(m-1) \sum_{j=1}^{N}\left[p_{0}\left(\mu_{\ell_{j}}\right)-p_{0}\left(\lambda_{j}\right)\right]}\left(e^{i \sum_{j=1}^{N}\left[p_{0}\left(\mu_{\ell_{j}}\right)-p_{0}\left(\lambda_{j}\right)\right]}-1\right) \\
& \times\left.\frac{\partial}{\partial \alpha} \frac{\left\langle\psi_{\kappa}(\{\mu\}) \mid \psi(\{\lambda\})\right\rangle}{\pi i\left\|\psi_{\kappa}(\{\mu\})\right\| \cdot\|\psi(\{\lambda\})\|}\right|_{\alpha=0},
\end{aligned}
$$

which is nonzero only if $N_{\kappa}=N$. The form-factor $\mathcal{F}_{\psi_{g} \psi^{\prime}}^{(z)}$ is obtained by complex conjugation $\mathcal{F}_{\psi_{g} \psi^{\prime}}^{(z)}(m)=\left(\mathcal{F}_{\psi^{\prime} \psi_{g}}^{(z)}(m)\right)^{*}$. Similarly, one obtains

$$
\begin{aligned}
& \mathcal{F}_{\psi^{\prime} \psi_{g}}^{(-)}(m)=\left.(-1)^{N+m+1} e^{i(m-1) \sum_{j=1}^{N_{\kappa}} p_{0}\left(\mu_{\ell_{j}}\right)-i m \sum_{j=1}^{N} p_{0}\left(\lambda_{j}\right)} \frac{\left\langle\psi_{\kappa}(\{\mu\})\left|B\left(-\frac{i \zeta}{2}\right)\right| \psi(\{\lambda\})\right\rangle}{a\left(-i \frac{\zeta}{2}\right)\left\|\psi_{\kappa}(\{\mu\})\right\| \cdot\|\psi(\{\lambda\})\|}\right|_{\alpha=0}, \\
& \mathcal{F}_{\psi_{g} \psi^{\prime}}^{(+)}(m)=\left.(-1)^{N+m} e^{i(m-1) \sum_{j=1}^{N} p_{0}\left(\lambda_{j}\right)-i m \sum_{j=1}^{N_{\kappa}} p_{0}\left(\mu_{\ell_{j}}\right)} \frac{\left\langle\psi(\{\lambda\})\left|C\left(-\frac{i \zeta}{2}\right)\right| \psi_{\kappa}(\{\mu\})\right\rangle}{a\left(-i \frac{\zeta}{2}\right)\left\|\psi_{\kappa}(\{\mu\})\right\| \cdot\|\psi(\{\lambda\})\|}\right|_{\alpha=0}
\end{aligned}
$$

which are nonzero only if $N_{\kappa}=N+1$. In (4.3) and (4.4), $a(\nu)=\sinh ^{M}(\nu-i \zeta / 2)$ denotes the eigenvalue of the operator $A(\nu)$ on the reference state which, in the case of the spin- $1 / 2$ chain, is the ferromagnetic state with all spins up. In the following, we will also use the notation $d(\nu)=\sinh ^{M}(\nu+i \zeta / 2)$ for the eigenvalue of $D(\nu)$ on this reference state.

Remark 4.1. Although it is possible to set $\alpha=0$ directly in (4.3) and (4.4), we prefer to take the limit $\alpha=0$ only in the very end of the calculations to study the properties of the scalar products for general $\alpha$. 
In all the above scalar products, one of the states is an eigenstate of the twisted transfer matrix. There exists an explicit determinant representations for such scalar products and the associated norms:

Proposition 4.1. Let $\{\lambda\}_{1}^{N}$ satisfy the ground state Bethe equations (2.2) and $\{\mu\}_{1}^{N_{\kappa}}$ solve the system of $\alpha$-twisted Bethe Ansatz equations (2.11). Then the following representations for the scalar products hold:

$$
\begin{aligned}
& \left\langle\psi_{\kappa}(\{\mu\}) \mid \psi(\{\lambda\})\right\rangle=\delta_{N_{\kappa}, N} \cdot \prod_{a, b=1}^{N} \frac{\sinh \left(\mu_{\ell_{a}}-\lambda_{b}-i \zeta\right)}{\sinh \left(\lambda_{a}-\mu_{\ell_{b}}\right)} \cdot \prod_{j=1}^{N}\left\{d\left(\mu_{\ell_{j}}\right) d\left(\lambda_{j}\right)\left[e^{2 i \pi \widehat{F}\left(\lambda_{j}\right)}-1\right]\right\} \\
& \quad \times \frac{1-\kappa}{1-e^{2 \pi i \widehat{F}(-q)}} \cdot \prod_{a=1}^{N} \frac{\sinh \left(q+\lambda_{a}-i \zeta\right)}{\sinh \left(q-\mu_{\ell_{a}}-i \zeta\right)} \cdot \operatorname{det}_{\Gamma_{q}}\left[I+\frac{1}{2 \pi i} \widehat{U}^{(z)}\left(w, w^{\prime}\right)\right], \quad(4.5) \\
& \left\langle\psi_{\kappa}(\{\mu\})|B(-i \zeta / 2)| \psi(\{\lambda\})\right\rangle=\delta_{N_{\kappa}, N+1} \cdot a(-i \zeta / 2) \sinh (-i \zeta) \prod_{j=1}^{N}\left\{a\left(\lambda_{j}\right)\left[1-e^{2 i \pi \widehat{F}\left(\lambda_{j}\right)}\right]\right\} \\
& \quad \times \frac{\prod_{b=1}^{N} \sinh \left(\lambda_{b}-i \zeta / 2\right)}{\prod_{b=1}^{N+1} \sinh \left(\mu_{\ell_{b}}+i \zeta / 2\right)} \prod_{a=1}^{N+1}\left\{d\left(\mu_{\ell_{a}}\right) \prod_{b=1}^{N} \frac{\sinh \left(\mu_{\ell_{a}}-\lambda_{b}-i \zeta\right)}{\sinh \left(\mu_{\ell_{a}}-\lambda_{b}\right)}\right\} \cdot \operatorname{det}\left[I+\frac{1}{\Gamma_{q}} \widehat{U}^{(+)}\left(w, w^{\prime}\right)\right] .
\end{aligned}
$$

In these expressions $\widehat{F}(\lambda)(2.13)$ depends on whether we consider the $N$ sector or the $(N+1)$ sector (see (2.12) ). The integral operators $I+\frac{1}{2 \pi i} \widehat{U}^{(z)}$ and $I+\frac{1}{2 \pi i} \widehat{U}^{(+)}$act on a closed anticlockwise oriented contour $\Gamma_{q}$ surrounding the interval $[-q, q]$ where the ground state roots $\left\{\lambda_{a}\right\}_{a=1}^{N}$ condensate and containing no other singularity of the kernels. The last ones have the form

$$
\begin{gathered}
\widehat{U}^{(z)}\left(w, w^{\prime}\right)=-\prod_{a=1}^{N} \frac{\sinh \left(w-\mu_{\ell_{a}}\right) \sinh \left(w-\lambda_{a}+i \zeta\right)}{\sinh \left(w-\lambda_{a}\right) \sinh \left(w-\mu_{\ell_{a}}+i \zeta\right)} \cdot \frac{K_{\kappa}\left(w-w^{\prime}\right)-K_{\kappa}\left(-q-w^{\prime}\right)}{1-e^{2 \pi i \widehat{F}(w)}}, \\
\widehat{U}^{(+)}\left(w, w^{\prime}\right)=\prod_{a=1}^{N+1} \frac{\sinh \left(w-\mu_{\ell_{a}}\right)}{\sinh \left(w-\mu_{\ell_{a}}+i \zeta\right)} \prod_{a=1}^{N} \frac{\sinh \left(w-\lambda_{a}+i \zeta\right)}{\sinh \left(w-\lambda_{a}\right)} \cdot \frac{\mathcal{K}_{\kappa}\left(w, w^{\prime}\right)}{1-e^{2 \pi i \widehat{F}(w)}},
\end{gathered}
$$

where $K_{\kappa}$ and $\mathcal{K}_{\kappa}$ are given respectively by (3.10) and (3.13).

The representation (4.5) was obtained in $[24,25,10,36$. The representation (4.6) is derived in appendix $\mathrm{B}$.

We also recall the finite size determinant representations for the norms of Bethe states: 
Proposition 4.2. [51, 52, 53] Let $\mu_{\ell_{1}}, \ldots, \mu_{\ell_{N_{\kappa}}}$ satisfy the system (2.12). Then

$$
\left\langle\psi_{\kappa}(\{\mu\}) \mid \psi_{\kappa}(\{\mu\})\right\rangle=(-1)^{N_{\kappa}} \prod_{j=1}^{N_{\kappa}}\left[2 \pi i M \widehat{\rho}_{\kappa}\left(\mu_{\ell_{j}}\right) a\left(\mu_{\ell_{j}}\right) d\left(\mu_{\ell_{j}}\right)\right] \frac{\prod_{a, b=1}^{N_{\kappa}} \sinh \left(\mu_{\ell_{a}}-\mu_{\ell_{b}}-i \zeta\right)}{\prod_{\substack{a, b=1 \\ a \neq b}}^{N_{\kappa}} \sinh \left(\mu_{\ell_{a}}-\mu_{\ell_{b}}\right)}
$$

where

$$
\Theta_{j k}^{(\mu)}=\delta_{j k}+\frac{K\left(\mu_{\ell_{j}}-\mu_{\ell_{k}}\right)}{2 \pi M \hat{\rho}_{\kappa}\left(\mu_{\ell_{k}}\right)} .
$$

One can see from another representation for the scalar product (B.1) that, when $\Im \alpha=0$,

$$
\left\langle\psi_{\kappa}(\{\mu\}) \mid \psi(\{\lambda\})\right\rangle=\left(\left\langle\psi_{\kappa}(\{\mu\}) \mid \psi(\{\lambda\})\right\rangle\right)^{*},
$$

and

$$
\left\langle\psi(\{\lambda\})\left|C\left(-\frac{i \zeta}{2}\right)\right| \psi_{\kappa}(\{\mu\})\right\rangle=\kappa^{-1} e^{i \sum_{j=1}^{N_{\kappa}} p_{0}\left(\mu_{\ell_{j}}\right)+i \sum_{j=1}^{N} p_{0}\left(\lambda_{j}\right)}\left(\left\langle\psi_{\kappa}(\{\mu\})\left|B\left(-\frac{i \zeta}{2}\right)\right| \psi(\{\lambda\})\right\rangle\right)^{*} .
$$

For the calculation of the two-point correlation functions $\left\langle\sigma_{m}^{z} \sigma_{m^{\prime}}^{z}\right\rangle$ (resp. $\left.\left\langle\sigma_{m}^{+} \sigma_{m^{\prime}}^{-}\right\rangle\right)$, we need actually to sum up the products $\mathcal{F}_{\psi_{g} \psi^{\prime}}^{(z)}(m) \mathcal{F}_{\psi^{\prime} \psi_{g}}^{(z)}\left(m^{\prime}\right)\left(\operatorname{resp} . \mathcal{F}_{\psi_{g} \psi^{\prime}}^{(+)}(m) \mathcal{F}_{\psi^{\prime} \psi_{g}}^{(-)}\left(m^{\prime}\right)\right)$ over all eigenstates $\left|\psi^{\prime}\right\rangle$. We have the following result:

Proposition 4.3. The products of two form factors can be written in terms of the former scalar products as

$$
\begin{gathered}
\mathcal{F}_{\psi_{g} \psi^{\prime}}^{(z)}\left(m^{\prime}\right) \cdot \mathcal{F}_{\psi^{\prime} \psi_{g}}^{(z)}(m)=\left.e^{i\left(m-m^{\prime}\right) \widehat{\mathcal{P}}_{e x}} \frac{2 \sin ^{2}\left(\frac{\widehat{\mathcal{P}}_{e x}}{2}\right)}{\pi^{2}} \cdot \frac{\partial^{2}}{\partial \alpha^{2}} S_{N}^{z}\right|_{\alpha=0}, \\
\mathcal{F}_{\psi_{g} \psi^{\prime}}^{(+)}\left(m^{\prime}\right) \cdot \mathcal{F}_{\psi^{\prime} \psi_{g}}^{(-)}(m)=\left.(-1)^{m-m^{\prime}} e^{i\left(m-m^{\prime}\right) \widehat{\mathcal{P}}_{e x}} \cdot S_{N}^{+}\right|_{\alpha=0},
\end{gathered}
$$

where

$$
\begin{gathered}
S_{N}^{z}=\left|\frac{\left\langle\psi_{\kappa}(\{\mu\}) \mid \psi(\{\lambda\})\right\rangle}{\left\|\psi_{\kappa}(\{\mu\})\right\| \cdot\|\psi(\{\lambda\})\|}\right|^{2}, \\
S_{N}^{+}=-\frac{e^{-2 \pi i \alpha}}{a^{2}(-i \zeta / 2)}\left|\frac{\left\langle\psi_{\kappa}(\{\mu\})\left|B\left(-\frac{i \zeta}{2}\right)\right| \psi(\{\lambda\})\right\rangle}{\left\|\psi_{\kappa}(\{\mu\})\right\| \cdot\|\psi(\{\lambda\})\|}\right|^{2} .
\end{gathered}
$$

In (4.13) and (4.14), $\widehat{\mathcal{P}}_{\text {ex }}$ denotes the relative excitation momentum of the excited states in the $N_{\kappa}$ sector in respect to the ground state in the $N$ sector,

$$
\widehat{\mathcal{P}}_{e x}=\sum_{j=1}^{N_{\kappa}} p_{0}\left(\mu_{\ell_{j}}\right)-\sum_{j=1}^{N} p_{0}\left(\lambda_{j}\right) .
$$

Remark 4.2. Here, we did not consider the product $\mathcal{F}_{\psi_{g} \psi^{\prime}}^{(-)}(m) \mathcal{F}_{\psi^{\prime} \psi_{g}}^{(+)}\left(m^{\prime}\right)$, since the equal-time correlation function $\left\langle\sigma_{m}^{-} \sigma_{m^{\prime}}^{+}\right\rangle$can be obtained from $\left\langle\sigma_{m}^{+} \sigma_{m^{\prime}}^{-}\right\rangle$by the replacement $m \rightarrow m^{\prime}$. 
The calculation of the thermodynamic limit of $\widehat{\mathcal{P}}_{e x}$ is a relatively simple problem. Indeed, it follows from (2.4), (2.12) that

$$
\frac{\widehat{\mathcal{P}}_{e x}}{2 \pi}=\alpha \frac{N_{\kappa}}{M}+\sum_{j=1}^{N_{\kappa}} \hat{\xi}_{\kappa}\left(\mu_{\ell_{j}}\right)-\sum_{j=1}^{N} \hat{\xi}\left(\lambda_{j}\right)+\frac{N^{2}+N-N_{\kappa}^{2}-N_{\kappa}}{2 M}=\alpha \frac{N_{\kappa}}{M}+\sum_{k=1}^{n}\left(\hat{\xi}_{\kappa}\left(\mu_{p_{k}}\right)-\hat{\xi}_{\kappa}\left(\mu_{h_{k}}\right)\right) .
$$

Here we have used the antisymmetry of the bare phase $\vartheta(-\lambda)=-\vartheta(\lambda)$ and $\hat{\xi}_{\kappa}\left(\mu_{\ell_{j}}\right)=\ell_{j} / M$, $\hat{\xi}\left(\lambda_{j}\right)=j / M$. Then, using (2.6) , we obtain that the thermodynamic limit $\mathcal{P}_{\text {ex }}$ of $\hat{\mathcal{P}}_{\text {ex }}$ is given by the expression (3.2).

The non-trivial problem is the computation of the thermodynamic limit of the scalar products $S_{N}^{z}$ and $S_{N}^{+}$. This problem will be treated in the remaining part of the article.

\section{$5 \quad$ Thermodynamic limit of $S_{N}^{z}$}

In the paper [1] we studied the thermodynamic limit of the scalar product $S_{N}^{z}$ in the particular case where the state $\left|\psi_{\kappa}(\{\mu\})\right\rangle$ was the $\alpha$-twisted ground state in the $N$ sector. In the general case, when the excited state contains $n$ particles and $n$ holes, the result also depends on their rapidities $\left\{\mu_{p}\right\}$ and $\left\{\mu_{h}\right\}$. As we have already announced, such dependence is not sufficient to characterize completely the thermodynamic limit of the form factor. Indeed, the latter decomposes into a product of a smooth and a discrete part. The smooth part can actually be completely described in terms of the rapidities of the particles and holes. However, for the description of the discrete part one should use also the integer numbers $\left\{p_{a}\right\}$ and $\left\{h_{a}\right\}$.

In the first part of this section, we explicitly factorize $S_{N}^{z}$ into the aforementioned product. Then, we investigate the thermodynamic limit of the smooth part, postponing the more complicated analysis of the discrete part until subsection 5.3.

Note that in this section we deal only with the excited states in the $N$ sector. Therefore the thermodynamic limit of the shift function $\widehat{F}(\lambda)$ is equal to $F^{(z)}(\lambda)$ given by (2.16). However, in order to lighten notations we omit the superscript $(z)$ throughout this section, denoting the limiting value of the shift function simply by $F(\lambda)$.

\subsection{Representation of the scalar product $S_{N}^{z}$}

Assume that the excited state $\left|\psi_{\kappa}(\{\mu\})\right\rangle$ is an excited state in the $N$ sector containing $n$ particles with rapidities $\left\{\mu_{p_{a}}\right\}_{a=1}^{n}$ and $n$ holes with rapidities $\left\{\mu_{h_{a}}\right\}_{a=1}^{n}$. One can fairly expect that the limiting value of $S_{N}^{z}$ depends on the thermodynamic limit of these rapidities $\left\{\mu_{p}\right\}$ and $\left\{\mu_{h}\right\}$, i.e. $\lim S_{N}^{z}=S_{n}^{z}\left(\left\{\mu_{p}\right\},\left\{\mu_{h}\right\}\right)$.

Using Propositions 4.1 and 4.2 as well as (4.15), it is readily seen that $S_{N}^{z}$ has the following representation:

$$
S_{N}^{z}\left(\left\{\mu_{p}\right\},\left\{\mu_{h}\right\}\right)=\mathcal{A}_{N}^{(z)}\left(\left\{\mu_{p}\right\},\left\{\mu_{h}\right\}\right) \cdot D_{N}^{(z)}\left(\left\{\mu_{p}\right\},\left\{\mu_{h}\right\}\right) \cdot \exp \left\{C_{N}^{(z)}\left(\left\{\mu_{p}\right\},\left\{\mu_{h}\right\}\right)\right\},
$$

where

$$
C_{N}^{(z)}\left(\left\{\mu_{p}\right\},\left\{\mu_{h}\right\}\right)=\sum_{a, b=1}^{N} \log \frac{\sinh \left(\lambda_{a}-\mu_{\ell_{b}}-i \zeta\right) \sinh \left(\mu_{\ell_{b}}-\lambda_{a}-i \zeta\right)}{\sinh \left(\lambda_{a}-\lambda_{b}-i \zeta\right) \sinh \left(\mu_{\ell_{a}}-\mu_{\ell_{b}}-i \zeta\right)}
$$




$$
D_{N}^{(z)}\left(\left\{\mu_{p}\right\},\left\{\mu_{h}\right\}\right)=\left(\operatorname{det}_{N} \frac{1}{\sinh \left(\mu_{\ell_{j}}-\lambda_{k}\right)}\right)^{2} \cdot \prod_{j=1}^{N} \frac{\sin ^{2} \pi \widehat{F}\left(\lambda_{j}\right)}{\pi^{2} M^{2} \widehat{\rho}\left(\lambda_{j}\right) \widehat{\rho}_{\kappa}\left(\mu_{\ell_{j}}\right)}
$$

and

$$
\mathcal{A}_{N}^{(z)}\left(\left\{\mu_{p}\right\},\left\{\mu_{h}\right\}\right)=\left|\frac{\sin \pi \alpha}{\sin \pi \widehat{F}(-q)} \prod_{a=1}^{N} \frac{\sinh \left(q+\lambda_{a}-i \zeta\right)}{\sinh \left(q-\mu_{\ell_{a}}-i \zeta\right)}\right|^{2} \frac{\left|\operatorname{det}_{\Gamma_{q}}\left[I+\frac{1}{2 \pi i} \widehat{U}^{(z)}\left(w, w^{\prime}\right)\right]\right|^{2}}{\operatorname{det}_{N} \Theta_{j k}^{(\lambda)} \cdot \operatorname{det}_{N} \Theta_{j k}^{(\mu)}} .
$$

We remind here that $\widehat{U}^{(z)}$ has been defined in (4.7) and that the $N \times N$ matrices $\Theta_{j k}^{(\lambda)}$ and $\Theta_{j k}^{(\mu)}$ are

$$
\Theta_{j k}^{(\lambda)}=\delta_{j k}+\frac{K\left(\lambda_{j}-\lambda_{k}\right)}{2 \pi M \widehat{\rho}\left(\lambda_{k}\right)}, \quad \Theta_{j k}^{(\mu)}=\delta_{j k}+\frac{K\left(\mu_{\ell_{j}}-\mu_{\ell_{k}}\right)}{2 \pi M \hat{\rho}_{k}\left(\mu_{\ell_{k}}\right)} .
$$

\subsection{Thermodynamic limit of the smooth part}

The smooth part of the scalar product consists of factors $\mathcal{A}_{N}^{(z)}$ and $C_{N}^{(z)}$. Their limits can be computed exactly in the same manner as in [1]. We set

$$
\begin{aligned}
& C_{n}^{(z)}\left(\left\{\mu_{p}\right\},\left\{\mu_{h}\right\}\right)=\lim _{N, M \rightarrow \infty} C_{N}^{(z)}\left(\left\{\mu_{p}\right\},\left\{\mu_{h}\right\}\right), \\
& \mathcal{A}_{n}^{(z)}\left(\left\{\mu_{p}\right\},\left\{\mu_{h}\right\}\right)=\lim _{N, M \rightarrow \infty} \mathcal{A}_{N}^{(z)}\left(\left\{\mu_{p}\right\},\left\{\mu_{h}\right\}\right) .
\end{aligned}
$$

We illustrate the main idea behind the calculation of $C_{n}^{(z)}$ and $\mathcal{A}_{n}^{(z)}$ on the following toyexample. Assume that $f(\lambda) \in C^{1}(\mathbb{R})$, and consider the computation of the thermodynamic limit of the sum

$$
S_{f}=\sum_{j=1}^{N}\left[f\left(\mu_{\ell_{j}}\right)-f\left(\lambda_{j}\right)\right] .
$$

To compute this limit one can decompose the sum as follows

$$
S_{f}=\sum_{j=1}^{N}\left[f\left(\mu_{j}\right)-f\left(\lambda_{j}\right)\right]+\sum_{j=1}^{n}\left[f\left(\mu_{p_{j}}\right)-f\left(\mu_{h_{j}}\right)\right] \rightarrow \sum_{j=1}^{n}\left[f\left(\mu_{p_{j}}\right)-f\left(\mu_{h_{j}}\right)\right]+\int_{-q}^{q} F(\lambda) f^{\prime}(\lambda) d \lambda .
$$

There we have used the leading behavior in $M^{-1}(2.14)$ for the spacing between $\mu_{j}$ and $\lambda_{j}$. It is convenient to introduce a modified shift function by

$$
F_{\text {mod }}(\lambda)=F(\lambda) \chi_{[-q, q]}(\lambda)+\sum_{j=1}^{n}\left(\chi_{]-\infty, \mu_{\left.p_{j}\right]}\right]}(\lambda)-\chi_{]-\infty, \mu_{h_{j}}\right]}(\lambda)\right),
$$

where $\chi_{[a, b]}$ is the characteristic function of the interval $[a, b]$. Then equation (5.8) can be recast simply as

$$
S_{f} \rightarrow \int_{-\infty}^{\infty} F_{\text {mod }}(\lambda) f^{\prime}(\lambda) d \lambda
$$

Therefore, provided that one extends the integration contours to the whole real axis and replaces $F$ with $F_{\text {mod }}$, one formally reduces the computations to the case considered in [1] where one 
was dealing with an excited state $\mid \psi_{\kappa}(\{\mu\}\rangle$ having no particles or holes. From this observation, the results of section 3 follow straightforwardly (by a mere replacement of the shift function by $\left.F_{m o d}\right)$ from those of [1]. In this way, starting from the equations (5.2), (5.4) we arrive at (3.5), (3.8).

Thus, the limits $\mathcal{A}_{n}^{(z)}$ and $C_{n}^{(z)}$ are well defined for arbitrary positions of particles and holes. They depend on $\left\{\mu_{p}\right\}$ and $\left\{\mu_{h}\right\}$ only. In particular, they do not depend on the underlying integer numbers.

At this stage it is interesting to consider the limiting case where, in the thermodynamic limit, all rapidities of particles and holes condensate on the Fermi boundaries.

Corollary 5.1. Let $\left|\psi_{\kappa}(\{\mu\})\right\rangle$ belongs to the $\mathbf{P}_{r}$ class as in (3.20), and denote

$$
\begin{aligned}
& C_{n, r}^{(z)}=C_{n}^{(z)}\left(\{+q\}_{n_{p}^{+}} \cup\{-q\}_{n_{p}^{-}},\{+q\}_{n_{h}^{+}} \cup\{-q\}_{n_{h}^{-}}\right), \\
& \mathcal{A}_{n, r}^{(z)}=\mathcal{A}_{n}^{(z)}\left(\{+q\}_{n_{p}^{+}} \cup\{-q\}_{n_{p}^{-}},\{+q\}_{n_{h}^{+}} \cup\{-q\}_{n_{h}^{-}}\right),
\end{aligned}
$$

where subscripts show the number of elements in the corresponding subsets. Let $F_{r}(\lambda)=F(\lambda)+r$

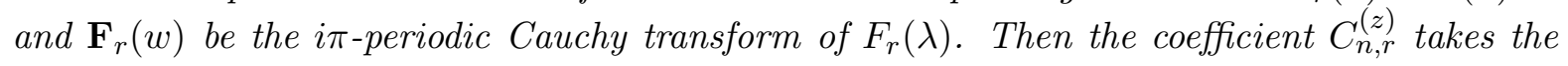
form

$$
C_{n, r}^{(z)}=C_{0}\left[F_{r}\right]
$$

where the functional $C_{0}[F]$ is defined in (3.6). The coefficient $\mathcal{A}_{n, r}^{(z)}$ becomes

$$
\mathcal{A}_{n, r}^{(z)}=\left(\frac{\sin \pi \alpha}{\sin \pi F(-q)}\right)^{2}\left|e^{-2 \pi i \mathbf{F}_{r}(-q+i \zeta)}\right|^{2}\left|\frac{\operatorname{det}\left[I+\frac{1}{2 \pi i} U_{r}^{(z)}\left(w, w^{\prime}\right)\right]}{\operatorname{det}\left[I+\frac{1}{2 \pi} K\right]}\right|^{2},
$$

where

$$
U_{r}^{(z)}\left(w, w^{\prime}\right)=-e^{2 \pi i\left(\mathbf{F}_{r}(w)-\mathbf{F}_{r}(w+i \zeta)\right)} \frac{K_{\kappa}\left(w-w^{\prime}\right)-K_{\kappa}\left(-q-w^{\prime}\right)}{1-e^{2 \pi i F(w)}} .
$$

Proof. This follows straightforwardly from $F_{\text {mod }}(\lambda)=F_{r}(\lambda) \chi_{[-q, q]}(\lambda)$.

Using (2.9), (2.16) we find that

$$
F_{r}(\lambda)=(r+\alpha) Z(\lambda)
$$

and we see that up to a replacement $\alpha+r \rightarrow \alpha$ our results coincide with the ones obtained in [1]. This coincidence is not accidental.

In the thermodynamic limit, the class $\mathbf{P}_{r}$ contains an infinite number of states. Indeed, knowing that a particle or hole's rapidity equals to $\pm q$ in this limit does not allow to fix the corresponding integers $p$ (resp. $h$ ) unambiguously. For instance, knowing that $\mu_{p}=q$ only allows one to say that $p / M \rightarrow D$. The latter is satisfied as long as one chooses $p=N+u_{M}$ with $u_{M}=\mathrm{o}(M)$, but arbitrary otherwise.

It is clear that quantities having well defined thermodynamic limits (such as $C_{n, r}^{(z)}$ and $\mathcal{A}_{n, r}^{(z)}$ ), must only depend on the macroscopic realization $\left\{\mu_{p}\right\}$ and $\left\{\mu_{h}\right\}$ of the excited state and not on the microscopic quantum numbers $\{p\}$ and $\{h\}$ leading to such a macroscopic configuration of the rapidities. Therefore, the calculation of their thermodynamic limit in the case of an excited state belonging to the $\mathbf{P}_{r}$ class can be done by choosing any of its representative. One 
of these representatives is given by the $(\alpha+r)$-twisted ground state. Indeed, set $n_{p}^{-}=n_{h}^{+}=0$ and choose the integers describing the holes and particles according to

$$
h_{k}=k, \quad p_{k}=N+k, \quad k=1, \ldots, r .
$$

The excited state is thus described by the set of integers,

$$
\ell_{j}=j \quad \text { for } \quad j=r+1, \ldots, N, \quad \text { and } \quad \ell_{j}=N+j \quad \text { for } \quad j=1, \ldots, r .
$$

Observe now that if we replace $\alpha$ by $\alpha+r$ in the r.h.s. of (2.11), then up to a re-ordering of the equations, we obtain the same set of integers (5.17). Thus, the above representative of the class $\mathbf{P}_{r}$ coincides with the $(\alpha+r)$-twisted ground state. Therefore the smooth part of all the critical form factors belonging to the $\mathbf{P}_{r}$ class coincides with the one of the form factor of the $\sigma^{z}$ operator taken between the ground state and the $(\alpha+r)$-twisted ground state.

\subsection{Thermodynamic limit of the discrete part}

As we have seen, the modified shift function (5.9) can be used for the calculation of the thermodynamic limits of sums (products, determinants) in the case when we deal with smooth functions of the rapidities of the excited state. One cannot apply this method in the case of the factor $D_{N}^{(z)}\left(\left\{\mu_{p}\right\},\left\{\lambda_{h}\right\}\right)$ as it depends on the Cauchy determinant of $\{\lambda\}$ and $\{\mu\}$. In the thermodynamic limit, certain entries of this matrix become divergent and more care is needed for the calculation of this limit.

Proposition 5.1. The discrete part $D_{N}^{(z)}$ behaves in the thermodynamic as

$$
\begin{aligned}
D_{N}^{(z)}\left(\left\{\mu_{p}\right\},\left\{\mu_{h}\right\}\right)=M^{-F_{+}^{2}-F_{-}^{2}} \cdot E_{0} & \left(\left\{\mu_{h}, \mu_{p}\right\} ;\left\{h_{a}, p_{a}\right\}\right) \cdot \prod_{k=1}^{n} H_{N}\left(\mu_{h_{k}}, h_{k}\right) \\
\times & \prod_{k=1}^{n} P_{N}\left(\mu_{p_{k}}, p_{k}\right) \cdot \mathcal{D}^{(z)}[F] \cdot\left(1+\mathrm{O}\left(\frac{\log M}{M}\right)\right) .
\end{aligned}
$$

The factor $E_{0}$ depends explicitly on the integers $\left\{h_{a}\right\}$ and $\left\{p_{a}\right\}$ which are the quantum numbers defining the excited state

$$
E_{0}=\frac{\prod_{\substack{j, k=1 \\ j \neq k}}^{n}\left(h_{j}-h_{k}\right)\left(p_{j}-p_{k}\right) \varphi\left(\mu_{h_{j}}, \mu_{h_{k}}\right) \varphi\left(\mu_{p_{j}}, \mu_{p_{k}}\right)}{\prod_{j, k=1}^{n}\left(p_{j}-h_{k}\right)^{2} \varphi^{2}\left(\mu_{p_{j}}, \mu_{h_{k}}\right)},
$$

where $\varphi(\lambda, \mu)=(2 \pi \sinh (\lambda-\mu)) /(p(\lambda)-p(\mu))$ and $p(\lambda)$ is the dressed momentum (2.6). The factors $P_{N}$ and $H_{N}$ also depend on the quantum numbers defining the holes and the particles

$$
\begin{gathered}
P_{N}\left(\mu_{p_{k}}, p_{k}\right)=\frac{e^{J\left[F^{(z)}\right]\left(\mu_{p_{k}}\right)}}{\rho\left(\mu_{p_{k}}\right)} \Gamma^{2}\left(\begin{array}{c}
p_{k}, p_{k}-N+F\left(\mu_{p_{k}}\right) \\
p_{k}+F\left(\mu_{p_{k}}\right), p_{k}-N
\end{array}\right), \\
H_{N}\left(\mu_{h_{k}}, h_{k}\right)=\frac{\sin ^{2}\left(\pi F\left(\mu_{h_{k}}\right)\right)}{e^{J\left[F^{(z)}\right]\left(\mu_{h_{k}}\right)} \pi^{2} \rho\left(\mu_{h_{k}}\right)} \Gamma^{2}\left(\begin{array}{c}
h_{k}+F\left(\mu_{h_{k}}\right), N+1-h_{k}-F\left(\mu_{h_{k}}\right) \\
h_{k}, N+1-h_{k}
\end{array}\right) .
\end{gathered}
$$


There we have introduced the functional $J$ which is defined in terms of the dressed momentum $p(\lambda)$ and acts on the shift function $F$ :

$$
J[F](\omega)=2 \int_{-q}^{q}\left\{F(\lambda) \partial_{\lambda} \log \varphi(\lambda, \omega)+\frac{F(\lambda)-F(\omega)}{p(\lambda)-p(\omega)} p^{\prime}(\lambda)\right\} d \lambda .
$$

Finally, we remind that the functional $\mathcal{D}^{(z)}$ is given in (3.16)

Remark 5.1. The definition of $P_{N}\left(\mu_{p_{k}}, p_{k}\right)$ is given in the case where the rapidities of all particles are to the right of the Fermi zone $\left(\right.$ ie $\left.p_{k}>N\right)$. If some particles have their rapidities to the left of the Fermi zone $\left(p_{k} \leq 0\right)$, then the corresponding arguments of the $\Gamma$-functions in (5.20) become negative integers. The aforementioned formula remains however valid provided that the arguments of these $\Gamma$-function are understood as limits

$$
\frac{\Gamma\left(p_{k}\right)}{\Gamma\left(p_{k}-N\right)}=\lim _{\varepsilon \rightarrow 0} \frac{\Gamma\left(p_{k}+\varepsilon\right)}{\Gamma\left(p_{k}-N+\varepsilon\right)}=(-1)^{N} \frac{\Gamma\left(N+1-p_{k}\right)}{\Gamma\left(1-p_{k}\right)} .
$$

Proof. Following the strategy applied in [1, we multiply and divide the original Cauchy determinant by the Cauchy determinant of the counting functions $\widehat{\xi}_{\kappa}(\lambda)$. Let

$$
\widehat{\varphi}(\lambda, \mu)=\frac{\sinh (\lambda-\mu)}{\widehat{\xi}(\lambda)-\widehat{\xi}(\mu)}, \quad \widehat{\varphi}_{\kappa}(\lambda, \mu)=\frac{\sinh (\lambda-\mu)}{\widehat{\xi}_{\kappa}(\lambda)-\widehat{\xi}_{\kappa}(\mu)} .
$$

After some algebra, we recast $D_{N}^{(z)}\left(\left\{\mu_{p}\right\},\left\{\lambda_{h}\right\}\right)$ into the following product:

$$
D_{N}^{(z)}\left(\left\{\mu_{p}\right\},\left\{\lambda_{h}\right\}\right)=\widehat{E}_{0} \cdot \prod_{k=1}^{n} \widehat{H}_{k} \cdot \prod_{k=1}^{n} \widehat{P}_{k} \cdot D_{N, 0} \cdot
$$

Here $\widehat{E}_{0}$ depends only on the particle/hole rapidities and the corresponding integers:

$$
\widehat{E}_{0}=\frac{\prod_{\substack{j, k=1 \\ j \neq k}}^{n}\left(h_{j}-h_{k}\right)\left(p_{j}-p_{k}\right) \widehat{\varphi}_{\kappa}\left(\mu_{h_{j}}, \mu_{h_{k}}\right) \widehat{\varphi}_{\kappa}\left(\mu_{p_{j}}, \mu_{p_{k}}\right)}{\prod_{j, k=1}^{n}\left(p_{j}-h_{k}\right)^{2} \widehat{\varphi}_{\kappa}^{2}\left(\mu_{p_{j}}, \mu_{h_{k}}\right)} .
$$

The factors $\widehat{P}_{k}$ depend on the rapidities $\mu_{p_{k}}$, integers $p_{k}$, the ground state parameters $\{\lambda\}$, and the roots $\mu_{j}$ such that $\widehat{\xi}_{\kappa}\left(\mu_{j}\right)=j / M, j=1, \ldots, N$. Their explicit representations are

$$
\widehat{P}_{k}=\frac{1}{\widehat{\rho}_{\kappa}\left(\mu_{p_{k}}\right)} \prod_{j=1}^{N}\left\{\frac{j-p_{k}-\widehat{F}\left(\mu_{p_{k}}\right)}{j-p_{k}-\widehat{F}\left(\lambda_{j}\right)} \cdot \frac{\widehat{\varphi}_{\kappa}\left(\mu_{p_{k}}, \mu_{j}\right)}{\widehat{\varphi}_{\kappa}\left(\mu_{p_{k}}, \lambda_{j}\right)}\right\}^{2} \Gamma^{2}\left(\begin{array}{c}
p_{k}, p_{k}-N+\widehat{F}\left(\mu_{p_{k}}\right) \\
p_{k}+\widehat{F}\left(\mu_{p_{k}}\right), p_{k}-N
\end{array}\right) .
$$

The representations for $\widehat{H}_{k}$ are similar to (5.27), but they depend on $\mu_{h_{k}}$ and $h_{k}$ :

$$
\begin{aligned}
& \widehat{H}_{k}=\frac{\sin ^{2}\left(\pi \widehat{F}\left(\mu_{h_{k}}\right)\right)}{\pi^{2} \widehat{\rho}_{\kappa}\left(\mu_{h_{k}}\right)} \prod_{j=1}^{N}\left\{\frac{j-h_{k}-\widehat{F}\left(\lambda_{j}\right)}{j-h_{k}-\widehat{F}\left(\mu_{h_{k}}\right)} \cdot \frac{\widehat{\varphi}_{\kappa}\left(\mu_{h_{k}}, \lambda_{j}\right)}{\widehat{\varphi}_{\kappa}\left(\mu_{h_{k}}, \mu_{j}\right)}\right\}^{2} \\
& \times \Gamma^{2}\left(\begin{array}{c}
h_{k}+\widehat{F}\left(\mu_{h_{k}}\right), N+1-h_{k}-\widehat{F}\left(\mu_{h_{k}}\right) \\
h_{k},
\end{array}\right) .
\end{aligned}
$$


Finally, $D_{N, 0}$ does not depend on the particle/hole rapidities and the corresponding integers

$$
\begin{aligned}
D_{N, 0}=\prod_{j=1}^{N} & \frac{\widehat{\rho}_{\kappa}\left(\lambda_{j}\right)}{\widehat{\rho}\left(\lambda_{j}\right)} \prod_{j, k=1}^{N} \frac{\widehat{\varphi}_{\kappa}\left(\lambda_{j}, \lambda_{k}\right) \widehat{\varphi}_{\kappa}\left(\mu_{j}, \mu_{k}\right)}{\widehat{\varphi}_{\kappa}^{2}\left(\mu_{j}, \lambda_{k}\right)} \\
& \times \prod_{j>k}^{N}\left(1-\frac{\widehat{F}\left(\lambda_{j}\right)-\widehat{F}\left(\lambda_{k}\right)}{j-k}\right)^{2} \prod_{\substack{j, k=1 \\
j \neq k}}^{N}\left(1-\frac{\widehat{F}\left(\lambda_{j}\right)}{j-k}\right)^{-2} \prod_{j=1}^{N}\left\{\frac{\sin \left(\pi \widehat{F}\left(\lambda_{j}\right)\right)}{\pi \widehat{F}\left(\lambda_{j}\right)}\right\}^{2}
\end{aligned}
$$

The fact that $\widehat{E}_{0} \rightarrow E_{0}$ follows from $\widehat{\varphi}_{\kappa} \rightarrow \varphi$. The calculation of the limit of the first product in (5.29) is based on the definition of the shift function (2.13):

$$
\begin{aligned}
\prod_{j=1}^{N} \frac{\widehat{\rho}_{\kappa}\left(\lambda_{j}\right)}{\widehat{\rho}\left(\lambda_{j}\right)}=\prod_{j=1}^{N}\left(1+\frac{\left(\widehat{\xi}_{\kappa}^{\prime}\left(\lambda_{j}\right)-\widehat{\xi}^{\prime}\left(\lambda_{j}\right)\right)}{\widehat{\rho}\left(\lambda_{j}\right)}\right) \\
=\prod_{j=1}^{N}\left(1-\frac{\widehat{F}^{\prime}\left(\lambda_{j}\right)}{M \widehat{\rho}\left(\lambda_{j}\right)}\right) \rightarrow \exp \left(-\int_{-q}^{q} F^{\prime}(\lambda) d \lambda\right)=e^{F_{-}-F_{+}} .
\end{aligned}
$$

The limit of the remaining part of $D_{N, 0}$ was computed in [1, what gives us

$$
D_{N, 0}=M^{-F_{+}^{2}-F_{-}^{2}} \cdot \mathcal{D}^{(z)}[F] \cdot\left(1+\mathrm{O}\left(\frac{\log M}{M}\right)\right) .
$$

Finally, the limits $\widehat{P}_{k} \rightarrow P_{N}\left(\mu_{p_{k}}, p_{k}\right)$ and $\widehat{H}_{k} \rightarrow H_{N}\left(\mu_{h_{k}}, h_{k}\right)$ follow from

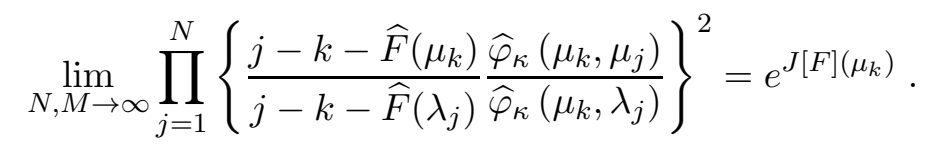

This formula is proved in appendix $\mathrm{A}$ (see (A.8)).

Assuming general positions of the particles and holes, we can not make further simplifications in the formula for $D_{N}^{(z)}$. Therefore we now study two limiting cases of interest, exactly as we did for the smooth parts: first when all particles and holes are separated from the Fermi boundaries, and then when all particles and holes are in the vicinity of the Fermi zone (critical form factor in the class $\mathbf{P}_{r}$ ).

Corollary 5.2. Suppose that, in the thermodynamic limit, particles and holes are separated from the Fermi boundaries: $\mu_{p_{a}} \neq \pm q$ and $\mu_{h_{a}} \neq \pm q$. Then the thermodynamic limit of the factor $D_{N}^{(z)}$ becomes a smooth function of the rapidities $\left\{\mu_{p}\right\}$ and $\left\{\mu_{h}\right\}$ :

$$
D_{N}^{(z)}=M^{-2 n-F_{-}^{2}-F_{+}^{2}} \cdot D_{n}^{(z)}\left(\left\{\mu_{p}\right\},\left\{\mu_{h}\right\}\right) \cdot\left(1+\mathrm{O}\left(\frac{\log M}{M}\right)\right)
$$

where $D_{n}^{(z)}\left(\left\{\mu_{p}\right\},\left\{\mu_{h}\right\}\right)$ is given by the expression (3.15) for $\mathcal{D}_{z z}$. 
Proof. If the rapidities of particles and holes are separated from the Fermi boundaries, then all the arguments of the $\Gamma$-functions in (5.20) and (5.21) are large and we can apply the Stirling formula for their simplification. Then

$$
\begin{gathered}
\lim _{N, M \rightarrow \infty} \Gamma^{2}\left(\begin{array}{rl}
p_{k}, p_{k}- & N+\widehat{F}\left(\mu_{p_{k}}\right), N+1-h_{k}-\widehat{F}\left(\mu_{h_{k}}\right), h_{k}+\widehat{F}\left(\mu_{h_{k}}\right) \\
& p_{k}-N, p_{k}+\widehat{F}\left(\mu_{p_{k}}\right), N+1-h_{k}, h_{k}
\end{array}\right) \\
=\lim _{N, M \rightarrow \infty}\left(\frac{p_{k}-N}{p_{k}}\right)^{2 F\left(\mu_{p_{k}}\right)}\left(\frac{N-h_{k}}{h_{k}}\right)^{-2 F\left(\mu_{h_{k}}\right)} \\
=\left(\frac{p\left(\mu_{p_{k}}\right)-p(q)}{p\left(\mu_{p_{k}}\right)-p(-q)}\right)^{2 F\left(\mu_{p_{k}}\right)}\left(\frac{p(q)-p\left(\mu_{h_{k}}\right)}{p\left(\mu_{h_{k}}\right)-p(-q)}\right)^{-2 F\left(\mu_{h_{k}}\right)}
\end{gathered}
$$

where we have used (2.6). Combining (5.34) with the explicit form (5.22) of $J(\omega)$, we arrive at

$$
\begin{aligned}
P_{N}\left(\mu_{p_{k}}, p_{k}\right) H_{N}\left(\mu_{h_{k}}, h_{k}\right)= & \frac{\sin ^{2}\left(\pi F\left(\mu_{h_{k}}\right)\right)}{\pi^{2} \rho\left(\mu_{h_{k}}\right) \rho\left(\mu_{p_{k}}\right)} \\
& \times \exp \left\{2 \int_{-q}^{q} F(\lambda)\left(\operatorname{coth}\left(\lambda-\mu_{p_{k}}\right)-\operatorname{coth}\left(\lambda-\mu_{h_{k}}\right)\right) d \lambda\right\} .
\end{aligned}
$$

The limit of $\widehat{E}_{0}$ is almost trivial:

$$
\lim _{N, M \rightarrow \infty} \widehat{E}_{0} M^{2 n}=\left(\operatorname{det}_{n} \frac{1}{\sinh \left(\mu_{p_{j}}-\mu_{h_{k}}\right)}\right)^{2} .
$$

Gathering all these results, we get the claim.

Hence, in the limit when the rapidities of particles and holes remain at finite distance from the Fermi boundaries, the discrete part $D_{N}^{(z)}$, up to an $M$-dependent normalization, can be approximated by a smooth function of these rapidities. However, as soon as some rapidities go to $\pm q$, then certain integrals in the second line of (3.15) become ill-defined. There can also appear singularities in the Cauchy determinants present in the first line of (3.15). Therefore, the discrete structure of $D_{N}^{(z)}$ manifests itself when particles and holes condensate on the Fermi boundaries.

We now describe the asymptotic behavior of an excited state $\mid \psi_{\kappa}\left(\left\{\mu_{\ell_{j}}\right\}\right\rangle$ belonging to the $\mathbf{P}_{r}$ class. This means that the rapidities of all particles and holes are located in vicinities of the Fermi boundaries with the condition (3.20). Any such state, can be parameterized by a set of integers as given in (3.21).

Corollary 5.3. The asymptotic behavior of $D_{N}^{(z)}$ for an excited state in the $\mathbf{P}_{r}$ class parameterized as in (3.21) reads

$$
D_{N}^{(z)}\left(\{+q\}_{n_{p}^{+}} \cup\{-q\}_{n_{p}^{-}},\{+q\}_{n_{h}^{+}} \cup\{-q\}_{n_{h}^{-}}\right)=M^{-F_{r,+}^{2}-F_{r,-}^{2}} D_{n, r}^{(z)}\left(1+\mathrm{O}\left(\frac{\log M}{M}\right)\right),
$$

where $D_{n, r}^{(z)}$ is given by the expression (3.23) for $\mathcal{D}_{z z}$. 
Remark 5.2. Thence, when particles and holes are in the vicinity of the Fermi boundaries, the behavior of $D_{N}^{(z)}$ cannot be called a thermodynamic limit. Indeed, the factors $R_{n_{p}^{+}, n_{h}^{+}}$and $R_{n_{p}^{-}, n_{h}^{-}}$, and thus the form factor, depend explicitly on the microscopic characteristics $p^{ \pm}$and $h^{ \pm}$of the excited state.

Proof. In this case, the limits $E_{0}, P_{N}\left(\mu_{p_{k}}, p_{k}\right)$ and $H_{N}\left(\mu_{h_{k}}, h_{k}\right)$ should be re-calculated.

Let us first consider the coefficient $\widehat{E}_{0}$. Using that $\varphi(q, q)=\varphi(-q,-q)=\rho^{-1}(q)$ and $\varphi(q,-q)=\varphi(-q, q)=\sinh (2 q) / D$, we get

$$
\lim _{N, M \rightarrow \infty} \widehat{E}_{0}=\left(\frac{D}{\rho(q) \sinh (2 q)}\right)^{2 r^{2}} \rho^{2 n}(q) \lim _{N, M \rightarrow \infty}\left(\operatorname{det} \frac{1}{p_{j}-h_{k}}\right)^{2} .
$$

Using the specific parametrization (3.21) of the integers belonging to the $\mathbf{P}_{r}$ class we see that the thermodynamic limit of the Cauchy determinant in (5.38) can be factorized in two parts:

$$
\left(\operatorname{det}_{n} \frac{1}{p_{j}-h_{k}}\right)^{2} \rightarrow N^{-2 r^{2}} L_{n_{p}^{+}, n_{h}^{+}}\left(\left\{p^{+}\right\},\left\{h^{+}\right\}\right) L_{n_{p}^{-}, n_{h}^{-}}\left(\left\{p^{-}\right\},\left\{h^{-}\right\}\right),
$$

with

$$
L_{n, m}(\{p\},\{h\})=\frac{\prod_{j>k}^{n}\left(p_{j}-p_{k}\right)^{2} \prod_{j>k}^{m}\left(h_{j}-h_{k}\right)^{2}}{\prod_{j=1}^{n} \prod_{k=1}^{m}\left(p_{j}+h_{k}-1\right)^{2}} .
$$

This leads eventually to the following estimate

$$
\lim _{N, M \rightarrow \infty} \widehat{E}_{0}(M \rho(q) \sinh (2 q))^{2 r^{2}}=\rho^{2 n}(q) L_{n_{p}^{+}, n_{h}^{+}}\left(\left\{p^{+}\right\},\left\{h^{+}\right\}\right) L_{n_{p}^{-}, n_{h}^{-}}\left(\left\{p^{-}\right\},\left\{h^{-}\right\}\right) .
$$

When computing the limits of $\widehat{P}_{k}$ and $\widehat{H}_{k}$, some of the arguments of the $\Gamma$-functions are not large anymore. Therefore, we can only use Stirling formula partly. We have

$$
\begin{gathered}
\prod_{k=1}^{n} \Gamma^{2}\left(\begin{array}{c}
p_{k}, p_{k}-N+\widehat{F}\left(\mu_{p_{k}}\right), N+1-h_{k}-\widehat{F}\left(\mu_{h_{k}}\right), h_{k}+\widehat{F}\left(\mu_{h_{k}}\right) \\
p_{k}-N, p_{k}+\widehat{F}\left(\mu_{p_{k}}\right), N+1-h_{k}, h_{k}
\end{array}\right) \\
\sim N^{-2 r\left(F_{+}+F_{-}\right)} \Gamma^{2}\left(\begin{array}{c}
\left\{p^{+}+F_{+}\right\},\left\{h^{+}-F_{+}\right\},\left\{h^{-}+F_{-}\right\},\left\{p^{-}-F_{-}\right\} \\
\left\{p^{+}\right\},\left\{h^{+}\right\},\left\{h^{-}\right\},\left\{p^{-}\right\}
\end{array}\right) .
\end{gathered}
$$

We precise that the notation $\left\{p^{+}+F_{+}\right\}$means $\left\{p_{j}^{+}+F_{+}\right\}_{j=1}^{n_{p}^{+}}$and similarly for other sets of parameters in (5.42). Combining all these results we obtain

$$
\begin{aligned}
\lim _{N, M \rightarrow \infty} \widehat{E}_{0}(M \rho(q) \sinh (2 q))^{2 r^{2}+2 r\left(F_{+}+F_{-}\right)} \prod_{k=1}^{n} \widehat{P}_{k} \widehat{H}_{k}= \\
\left(\frac{\sin \left(\pi F_{+}\right)}{\pi}\right)^{2 n_{h}^{+}}\left(\frac{\sin \left(\pi F_{-}\right)}{\pi}\right)^{2 n_{h}^{-}} R_{n_{p}^{+}, n_{h}^{+}}\left(\left\{p^{+}\right\},\left\{h^{+}\right\} \mid F_{+}\right) R_{n_{p}^{-}, n_{h}^{-}}\left(\left\{p^{-}\right\},\left\{h^{-}\right\} \mid-F_{-}\right) \\
\times \exp \left\{2 r \int_{-q}^{q} \frac{F_{+}-F(\lambda)}{\tanh (q-\lambda)} d \lambda+2 r \int_{-q}^{q} \frac{F_{-}-F(\lambda)}{\tanh (q+\lambda)} d \lambda\right\} .
\end{aligned}
$$

After some simple algebra we get the claim. 
To conclude this section we would like to stress that the evaluation of the $S_{N}^{z}$ thermodynamic limit was done without using the explicit form of the shift function $F(\lambda)(2.16)$. We have used this representation only in (5.15) in order to relate the smooth part of the critical form factors of $\mathbf{P}_{r}$ class with the one corresponding to the $(\alpha+r)$-twisted ground state. However in all other respects $F(\lambda)$ played the role of a free functional parameter.

\section{$6 \quad$ The scalar product $S_{N}^{+}$}

We now study the scalar product $S_{N}^{+}$for $\left|\psi_{\kappa}(\{\mu\})\right\rangle$ being an $\alpha$-twisted excited state with $n$ particles and $n$ holes in the $N+1$ sector (i.e. $N_{\kappa}=N+1$ ). As previously, $\left\{\mu_{p_{a}}\right\}_{a=1}^{n}$ and $\left\{\mu_{h_{a}}\right\}_{a=1}^{n}$ denote the rapidities of the particles and holes respectively. The notation $\widehat{F}(\lambda)$ now means the shift function in the $N+1$ sector, whose thermodynamic limit $F^{(+)}(\lambda)$ is given by (2.17). However, like in the previous section, we omit the superscript $(+)$ in order to lighten the notations.

\subsection{Representation for the scalar product $S_{N}^{+}$}

In comparison with the $S_{N}^{z}$ case, the excited state now depends on $N+1$ parameters $\mu$. Using the determinant representation given in Proposition 4.1 as well as the norm formula of Proposition 4.2, we get the following representation for $S_{N}^{+}(4.16)$ :

$$
S_{N}^{+}\left(\left\{\mu_{p}\right\},\left\{\mu_{h}\right\}\right)=\mathcal{A}_{N}^{(+)}\left(\left\{\mu_{p}\right\},\left\{\mu_{h}\right\}\right) \cdot \exp \left\{C_{N}^{(+)}\left(\left\{\mu_{p}\right\},\left\{\mu_{h}\right\}\right)\right\} \cdot D_{N}^{(+)}\left(\left\{\mu_{p}\right\},\left\{\mu_{h}\right\}\right) .
$$

Here

$$
D_{N}^{(+)}\left(\left\{\mu_{p}\right\},\left\{\mu_{h}\right\}\right)=\frac{M \pi^{2} \widehat{\rho}\left(\lambda_{N+1}\right)}{\sin ^{2} \pi \widehat{F}\left(\lambda_{N+1}\right)} \frac{\prod_{a=1}^{N+1} \sinh ^{2}\left(\lambda_{N+1}-\mu_{\ell_{a}}\right)}{\prod_{a=1}^{N} \sinh ^{2}\left(\lambda_{N+1}-\lambda_{a}\right)} \cdot D_{N+1}^{(z)}\left(\left\{\mu_{p}\right\},\left\{\mu_{h}\right\}\right),
$$

where $D_{N+1}^{(z)}$ is given by (5.3) with $N$ replaced by $N+1$. It is expressed in terms of the $N+1$ parameters $\mu_{\ell_{a}}$ as well as the $N+1$ parameters $\lambda_{j}$ defined by $\widehat{\xi}\left(\lambda_{j}\right)=j / M, \widehat{\xi}(\lambda)$ being the counting function (2.4) for the ground state. In other words $\lambda_{j}, j=1, \ldots, N$, are Bethe roots for the ground state, whereas $\lambda_{N+1}$ is the point where $M \widehat{\xi}(\lambda)$ takes its next integer value, ie $M \widehat{\xi}\left(\lambda_{N+1}\right)=N+1$.

The coefficient $C_{N}^{(+)}$is now modified according to

$$
\exp \left[C_{N}^{(+)}\left(\left\{\mu_{p}\right\},\left\{\mu_{h}\right\}\right)\right]=\prod_{a=1}^{N+1}\left|\frac{\sinh \left(\lambda_{a}-\lambda_{N+1}-i \zeta\right)}{\sinh \left(\mu_{\ell_{a}}-\lambda_{N+1}-i \zeta\right)}\right|^{2} \cdot \exp \left[C_{N+1}^{(z)}\left(\left\{\mu_{p}\right\},\left\{\mu_{h}\right\}\right)\right],
$$

where, up to the evident modifications stemming from $N \rightarrow N+1, C_{N+1}^{(z)}$ is given by (5.2). Finally the factor $\mathcal{A}_{N}^{(+)}$has the following form:

$$
\mathcal{A}_{N}^{(+)}\left(\left\{\mu_{p}\right\},\left\{\mu_{h}\right\}\right)=\frac{\sin \zeta}{2 \pi \kappa}\left|\frac{\prod_{a=1}^{N} \sinh \left(\lambda_{a}-\frac{i \zeta}{2}\right)}{\prod_{a=1}^{N+1} \sinh \left(\mu_{\ell_{a}}-\frac{i \zeta}{2}\right)}\right|^{2} \cdot \frac{\left|\operatorname{det}\left[I+\frac{1}{2 \pi i} \widehat{U}^{(+)}\left(w, w^{\prime}\right)\right]\right|^{2}}{\operatorname{det}_{N+1} \Theta_{j k}^{(\mu)} \cdot \operatorname{det}_{N} \Theta_{j k}^{(\lambda)}},
$$


and we refer to (4.8) for the definition of the kernel $\widehat{U}^{(+)}\left(w, w^{\prime}\right)$.

\subsection{Thermodynamic limit of the smooth part}

The smooth part of $S_{N}^{+}$consists of the coefficients $\mathcal{A}_{N}^{(+)}$and $\exp C_{N}^{(+)}$. The computation of their thermodynamic limits $C_{n}^{(+)}$and $\mathcal{A}_{n}^{(+)}$does not contain any subtleties comparing with the derivation described in section 5.2. The use of the modified shift function (5.9) formally reduces the computations to the case where the excited state does not contain particles and holes. In this way, starting from the representations (6.3), (6.4) we arrive at the equations (3.7), (3.11). One can easily see that the coefficients $C_{n}^{(+)}$and $\mathcal{A}_{n}^{(+)}$are well defined for general positions of particles and holes. In particular, for the critical form factors of the $\mathbf{P}_{r}$ class the smooth part effectively depends on the shift function $F_{r}(\lambda)=F(\lambda)+r$.

\subsection{Thermodynamic limit of the discrete part}

Proposition 6.1. The discrete part $D_{N}^{(+)}$behaves in the thermodynamic as

$$
\begin{aligned}
D_{N}^{(+)}\left(\left\{\mu_{p}\right\},\left\{\mu_{h}\right\}\right)=M^{-F_{-}^{2}-\left(F_{+}+1\right)^{2}} & E_{0}\left(\left\{\mu_{h}, \mu_{p}\right\},\left\{h_{a}, p_{a}\right\}\right) \cdot \prod_{k=1}^{n} \widetilde{H}_{N+1}\left(\mu_{h_{k}}, h_{k}\right) \\
\times & \prod_{k=1}^{n} \widetilde{P}_{N+1}\left(\mu_{p_{k}}, p_{k}\right) \cdot \mathcal{D}^{(+)}[F] \cdot\left(1+\mathrm{O}\left(\frac{\log M}{M}\right)\right),
\end{aligned}
$$

where

$$
\begin{aligned}
\widetilde{H}_{N+1}\left(\mu_{h_{k}}, h_{k}\right) & =H_{N+1}\left(\mu_{p_{k}}, p_{k}\right)\left(N+1-h_{k}-F\left(\mu_{h_{k}}\right)\right)^{-2} \varphi^{-2}\left(q, \mu_{h_{k}}\right), \\
\widetilde{P}_{N+1}\left(\mu_{p_{k}}, p_{k}\right) & =P_{N+1}\left(\mu_{p_{k}}, p_{k}\right)\left(N+1-p_{k}-F\left(\mu_{p_{k}}\right)\right)^{2} \varphi^{2}\left(q, \mu_{p_{k}}\right) .
\end{aligned}
$$

The functions entering these definitions are the same as in Proposition 5.1, but one should replace $N$ by $N+1$ for the coefficients $H_{N+1}$ and $P_{N+1}$. We also remind that the functional $\mathcal{D}^{(+)}$is given in (3.19).

Proof. As the limit of $D_{N+1}^{(z)}$ is already known, it remains to evaluate the limit of the product

$$
P=\frac{\prod_{a=1}^{N+1} \sinh ^{2}\left(\lambda_{N+1}-\mu_{\ell_{a}}\right)}{\prod_{a=1}^{N} \sinh ^{2}\left(\lambda_{N+1}-\lambda_{a}\right)}
$$

Harping on the steps in the analysis of $D_{N}^{(z)}$, we multiply and divide by the counting function $\widehat{\xi}(\lambda)(2.4)$. We obtain

$$
P=\frac{\prod_{a=1}^{N+1}\left(N+1-\ell_{a}-\widehat{F}\left(\mu_{\ell_{a}}\right)\right)^{2}}{M^{2} \prod_{a=1}^{N}(N+1-a)^{2}} \cdot \frac{\prod_{a=1}^{N+1} \widehat{\varphi}^{2}\left(\lambda_{N+1}, \mu_{\ell_{a}}\right)}{\prod_{a=1}^{N} \widehat{\varphi}^{2}\left(\lambda_{N+1}, \lambda_{a}\right)}
$$


Then using Corollary A.1 we find that, in the thermodynamic limit, the product $P$ behaves as

$$
P \rightarrow \frac{e^{J[F](q)}}{M^{2} \rho^{2}(q)} \Gamma^{2}\left(\begin{array}{c}
N+1-F_{+} \\
N+1,-F_{+}
\end{array}\right) \cdot \prod_{k=1}^{n}\left[\frac{N+1-p_{k}-F\left(\mu_{p_{k}}\right)}{N+1-h_{k}-F\left(\mu_{h_{k}}\right)} \cdot \frac{\varphi\left(q, \mu_{p_{k}}\right)}{\varphi\left(q, \mu_{h_{k}}\right)}\right]^{2},
$$

with $J[F](q)$ defined as in (5.22). Using now that $N$ is large, we have

$$
e^{J[F](q)} \Gamma^{2}\left(\begin{array}{c}
N+1-F_{+} \\
N+1,-F_{+}
\end{array}\right) \rightarrow \frac{\Gamma^{2}\left(1+F_{+}\right) \sin ^{2} \pi F_{+}}{\pi^{2}(\rho(q) \sinh (2 q) M)^{2 F_{+}}} \exp \left\{2 \int_{-q}^{q} \frac{F(\lambda)-F_{+}}{\tanh (\lambda-q)}\right\} .
$$

Taking into account (3.16), (3.19) we find

$$
\mathcal{D}^{(z)}[F] \cdot P \cdot \frac{M \pi^{2} \widehat{\rho}\left(\lambda_{N+1}\right)}{\sin ^{2}\left(\pi \widehat{F}\left(\lambda_{N+1}\right)\right)} \rightarrow \frac{\mathcal{D}^{(+)}[F]}{M^{2 F_{+}+1}} \prod_{k=1}^{n}\left[\frac{N+1-p_{k}-F\left(\mu_{p_{k}}\right)}{N+1-h_{k}-F\left(\mu_{h_{k}}\right)} \cdot \frac{\varphi\left(q, \mu_{p_{k}}\right)}{\varphi\left(q, \mu_{h_{k}}\right)}\right]^{2} .
$$

It remains to combine the obtained result with the known limit of $D_{N+1}^{(z)}$ and to substitute it into (6.2). A few algebraic manipulations lead then to the claim.

We now particularize this result to the two limiting cases we considered previously, namely when all particles and holes are separated from the Fermi boundaries, or when all particles and holes are on the Fermi boundaries.

Corollary 6.1. Suppose that, in the thermodynamic limit, particles and holes are separated from the Fermi boundaries: $\mu_{p_{a}} \neq \pm q$ and $\mu_{h_{a}} \neq \pm q$. Then the thermodynamic limit of $D_{N}^{(+)}$ becomes a smooth function of the rapidities $\left\{\mu_{p}\right\}$ and $\left\{\mu_{h}\right\}$ :

$$
D_{N}^{(+)}=M^{-2 n-F_{-}^{2}-\left(F_{+}+1\right)^{2}} \cdot D_{n}^{(+)}\left(\left\{\mu_{p}\right\},\left\{\mu_{h}\right\}\right) \cdot\left(1+\mathrm{O}\left(\frac{\log M}{M}\right)\right)
$$

where $D_{n}^{(+)}\left(\left\{\mu_{p}\right\},\left\{\mu_{h}\right\}\right)$ is given by the expression (3.18) for $\mathcal{D}_{+-}$.

Proof. If the rapidities of particles and holes are separated from the Fermi boundaries, then

$$
\prod_{k=1}^{n}\left[\frac{N+1-p_{k}-F\left(\mu_{p_{k}}\right)}{N+1-h_{k}-F\left(\mu_{h_{k}}\right)} \cdot \frac{\varphi\left(q, \mu_{p_{k}}\right)}{\varphi\left(q, \mu_{h_{k}}\right)}\right]^{2} \rightarrow \prod_{k=1}^{n}\left(\frac{\sinh \left(q-\mu_{p_{k}}\right)}{\sinh \left(q-\mu_{h_{k}}\right)}\right)^{2} .
$$

Substituting this limit into (6.5) and using the results of the previous section we arrive at the statement.

Corollary 6.2. The asymptotic behavior of $D_{N}^{(+)}$for an excited state in the $\mathbf{P}_{r}$ class parameterized as in (3.21) reads

$$
D_{N}^{(+)}\left(\{+q\}_{n_{p}^{+}} \cup\{-q\}_{n_{p}^{-}},\{+q\}_{n_{h}^{+}} \cup\{-q\}_{n_{h}^{-}}\right)=M^{-\left(F_{r,+}+1\right)^{2}-F_{r,-}^{2}} D_{n, r}^{(+)}\left(1+\mathrm{O}\left(\frac{\log M}{M}\right)\right),
$$

where $D_{n, r}^{(+)}$is given by the expression (3.24) for $\mathcal{D}_{+-}$. 
Proof. In this case

$$
\prod_{k=1}^{n}\left[\frac{N+1-p_{k}-F\left(\mu_{p_{k}}\right)}{N+1-h_{k}-F\left(\mu_{h_{k}}\right)} \cdot \frac{\varphi\left(q, \mu_{p_{k}}\right)}{\varphi\left(q, \mu_{h_{k}}\right)}\right]^{2} \rightarrow(M \rho(q) \sinh (2 q))^{-2 r} \frac{\prod_{k=1}^{n_{p}^{+}}\left(p_{k}^{+}+F_{+}\right)^{2}}{\prod_{k=1}^{n_{h}^{+}}\left(h_{k}^{+}-1-F_{+}\right)^{2}} .
$$

Substituting this limit into (6.5) and using the results of the previous section we arrive at the statement.

\section{Conclusion}

In this article we have provided the first steps towards the study of correlation functions via the form factor approach. For this purpose we have calculated the thermodynamic limits of the so-called particle/hole form factors. Although we have considered the specific case of the XXZ chain, our method is straightforwardly applicable to other massless integrable models solvable by means of the algebraic Bethe Ansatz and having determinant representations of their form factors. In particular, one can use it for the calculation of the thermodynamic limit of form factors in the model of one-dimensional bosons. Determinant representations for the form factors of this model in the finite volume were given in [19, 20]. Their thermodynamic limit can be directly obtained from the results given in the present article.

Our results show that the idea to replace, in the thermodynamic limit, the sum over excited states by an integration with respect to particles and holes should be essentially modified. In particular, a formal replacement of the discrete sums over $n$-particle/hole excited states $\left|\psi^{\prime}\left(\left\{\mu_{p}\right\},\left\{\mu_{h}\right\}\right)\right\rangle$ by integrals as

$$
\sum_{\left|\psi^{\prime}\left(\left\{\mu_{p}\right\},\left\{\mu_{h}\right\}\right)\right\rangle} \mathcal{F}_{\psi_{g} \psi^{\prime}}^{(s)}(m) \mathcal{F}_{\psi^{\prime} \psi_{g}}^{\left(s^{\prime}\right)}\left(m^{\prime}\right) \rightarrow M^{2 n} \int \mathcal{F}_{\psi_{g} \psi^{\prime}}^{(s)}(m) \mathcal{F}_{\psi^{\prime} \psi_{g}}^{\left(s^{\prime}\right)}\left(m^{\prime}\right) \prod_{j=1}^{n} \rho\left(\mu_{p_{j}}\right) \rho\left(\mu_{h_{j}}\right) d \mu_{p_{j}} d \mu_{h_{j}},
$$

leads to senseless results. First of all, even in the region where the form factors depend smoothly on $\left\{\mu_{p}\right\}$ and $\left\{\mu_{h}\right\}$, the coefficient $M^{2 n}$ does not compensate the factors $M^{-\theta_{s s^{\prime}}}$, therefore in the above expression, the r.h.s. vanishes. On the other hand, the corresponding integral becomes divergent when the rapidities of particles and holes approach the Fermi boundaries (see (3.15), (3.18) ). Lastly, due to the discrete structure of the form factors when some rapidities agglomerate on the Fermi boundaries, one cannot replace the sum over the excited states in the $\mathbf{P}_{r}$ class by an integral. For such excited states, one has to take the microscopic structure of the excited state into account and perform the discrete sums over the parameters $p^{ \pm}, h^{ \pm}$ (3.21).

In a forthcoming publication [54], we will describe a way to overcome these difficulties. We will show that in the asymptotic regime (large lattice distances $m$ between local spin opertors) only particles and holes having their rapidities close to the Fermi boundaries contribute to the form factor sums. In such a limit, one can send the rapidities of the particles and holes to $\pm q$ in the smooth part of the form factor. Hence, it becomes a constant that only depends on the $\mathbf{P}_{r}$ class $\mathcal{S}_{s s^{\prime}}=\mathcal{S}_{s s^{\prime}}^{(r)}(\{ \pm q\},\{ \pm q\})$. In its turn the summation over the integers $\{p\}$ and $\{h\}$ present in the discrete part of the form factors leads to the natural re-scaling $M \rightarrow 2 \pi m$ of the system 
size $M$ into the distance $m$ between the operators. This mechanism explains the appearance of the critical exponents $\theta_{s s^{\prime}}$ in the asymptotic behavior of the correlation functions.

\section{Acknowledgements}

J. M. M., N. S. and V. T. are supported by CNRS. We also acknowledge the support from the GDRI-471 of CNRS "French-Russian network in Theoretical and Mathematical Physics" and RFBR-CNRS-09-01-93106L-a. N. K., J. M. M. and V. T are also supported by the ANR grant DIADEMS 10 BLAN 012004 and N. S. by the Program of RAS Mathematical Methods of the Nonlinear Dynamics, RFBR-11-01-00440-a. K. K. K. is supported by the EU Marie-Curie Excellence Grant MEXT-CT-2006-042695. N. K., N. S. and K. K. K would like to thank the Theoretical Physics group of the Laboratory of Physics at ENS Lyon for hospitality, which makes this collaboration possible. N.K. and V. T. would like to thank LPTHE (Paris VI University) for hospitality.

\section{A Summation identities}

Lemma A.1. Let $f \in C^{1}([0, a])$ for some $a>D$. Let $\left(h_{M}\right)$ be a sequence of integers such that $\frac{h_{M}}{M}$ tends to some finite value $x_{h} \in[0, a]$ when $M \rightarrow+\infty$. Then, in the limit $N, M \rightarrow+\infty$, $N / M \rightarrow D$, the following sums vanish:

$$
\lim _{N, M \rightarrow \infty} \sum_{\substack{k=1 \\ k \neq h_{M}}}^{N}\left|\frac{f\left(\frac{k}{M}\right)-f\left(\frac{h_{M}}{M}\right)}{\left(k-h_{M}\right)^{n}}\right|=0, \quad n \geq 2 .
$$

Proof. Let $n=2$. Then

$$
\sum_{\substack{k=1 \\ k \neq h_{M}}}^{N} \frac{\left|f\left(\frac{k}{M}\right)-f\left(\frac{h_{M}}{M}\right)\right|}{\left(k-h_{M}\right)^{2}} \leq \frac{1}{M^{2}} \sum_{\substack{k=1 \\ k \neq h_{M}}}^{N} \frac{\left|f\left(\frac{k}{M}\right)-f\left(\frac{h_{M}}{M}\right)-\frac{k-h_{M}}{M} f^{\prime}\left(\frac{h_{M}}{M}\right)\right|}{\left(\frac{k-h_{M}}{M}\right)^{2}}+\frac{\left|f^{\prime}\left(\frac{h_{M}}{M}\right)\right|}{M} \sum_{\substack{k=1 \\ k \neq h_{M}}}^{N} \frac{1}{k-h_{M}} .
$$

The second term vanishes in the limit, as it is of order $\frac{\log N}{M}$. The first term vanishes due to the Euler-Maclaurin summation formula:

$$
\begin{aligned}
\lim _{\substack{N, M \rightarrow \infty \\
N / M \rightarrow D}} \frac{1}{M^{2}} \sum_{\substack{k=1 \\
k \neq h_{M}}}^{N} \frac{\left|f\left(\frac{k}{M}\right)-f\left(\frac{h_{M}}{M}\right)-\frac{k-h_{M}}{M} f^{\prime}\left(\frac{h_{M}}{M}\right)\right|}{\left(\frac{k-h_{M}}{M}\right)^{2}} \\
=\lim _{\substack{N, M \rightarrow \infty \\
N / M \rightarrow D}} \frac{1}{M} \int_{0}^{D} \frac{\left|f(x)-f\left(x_{h}\right)-\left(x-x_{h}\right) f^{\prime}\left(x_{h}\right)\right|}{\left(x-x_{h}\right)^{2}} d x=0 .
\end{aligned}
$$

For $n>2$, we have

$$
\sum_{\substack{k=1 \\ k \neq h_{M}}}^{N}\left|\frac{f\left(\frac{k}{M}\right)-f\left(\frac{h_{M}}{M}\right)}{\left(k-h_{M}\right)^{n}}\right|=\sum_{\substack{k=1 \\ k \neq h_{M}}}^{N}\left|\frac{1}{\left(k-h_{M}\right)^{n-2}} \cdot \frac{f\left(\frac{k}{M}\right)-f\left(\frac{h_{M}}{M}\right)}{\left(k-h_{M}\right)^{2}}\right| \leq \sum_{\substack{k=1 \\ k \neq h_{M}}}^{N} \frac{\left|f\left(\frac{k}{M}\right)-f\left(\frac{h_{M}}{M}\right)\right|}{\left(k-h_{M}\right)^{2}} \rightarrow 0,
$$

which ends the proof. 
Lemma A.2. With the hypothesis of Lemma A.1, let $n_{0} \in \mathbb{N}$ be such that $\sup _{x \in[0, D]}|f(x)|<n_{0}$ and $\left|f\left(x_{h}\right)\right|<n_{0}$. Then

$$
S_{n_{0} ; N}(f)=\sum_{\substack{k=1 \\\left|k-h_{M}\right| \geq n_{0}}}^{N} \log \frac{k-h_{M}+f\left(\frac{k}{M}\right)}{k-h_{M}+f\left(\frac{h_{M}}{M}\right)} \underset{\substack{N, M \rightarrow \infty \\ N / M \rightarrow D}}{\longrightarrow} \int_{0}^{D} \frac{f(x)-f\left(x_{h}\right)}{x-x_{h}} d x .
$$

Proof. Expanding the logarithms into their Taylor series we obtain

$$
S_{n_{0} ; N}(f)=\sum_{\substack{k=1 \\\left|k-h_{M}\right| \geq n_{0}}}^{N} \sum_{r=1}^{\infty} \frac{(-1)^{r+1}}{r} \cdot \frac{f^{r}\left(\frac{k}{M}\right)-f^{r}\left(\frac{h_{M}}{M}\right)}{\left(k-h_{M}\right)^{r}} .
$$

The result of Lemma A.1 shows that only the terms corresponding to $r=1$ give non-vanishing contributions. These are computed as a Riemann sum:

$$
\lim _{\substack{N, M \rightarrow \infty \\ N / M \rightarrow D}} S_{n_{0} ; N}(f)=\lim _{\substack{N, M \rightarrow \infty \\ N / M \rightarrow D}} \frac{1}{M} \sum_{\substack{k=1 \\\left|k-h_{M}\right| \geq n_{0}}}^{N} \frac{f\left(\frac{k}{M}\right)-f\left(\frac{h_{M}}{M}\right)}{\frac{k-h_{M}}{M}}=\int_{0}^{D} \frac{f(x)-f\left(x_{h}\right)}{x-x_{h}} d x .
$$

Corollary A.1. Let $f$ and $\left(h_{M}\right)$ satisfy the hypothesis of Lemma A.1. Let $\xi(\lambda)$ be a strictly monotonous smooth function on $\mathbb{R}$ such that $\xi^{-1}(0)=-q$ and $\xi(D)=q$. Define $\lambda_{k}=$ $\xi^{-1}(k / M), k \in \mathbb{Z}$ and $\rho(\lambda)=\xi^{\prime}(\lambda)$. Then, independently whether the thermodynamic limit $\lambda_{h}$ of $\lambda_{h_{M}}$ belongs or not to the interval $[-q, q]$, one has

$$
\lim _{\substack{N, M \rightarrow \infty \\ N / M \rightarrow D}} \prod_{k=1}^{N} \frac{k-h_{M}+f\left(\lambda_{k}\right)}{k-h_{M}+f\left(\lambda_{h_{M}}\right)}=\exp \left\{\int_{-q}^{q} \frac{f(\lambda)-f\left(\lambda_{h}\right)}{\xi(\lambda)-\xi\left(\lambda_{h}\right)} \rho(\lambda) d \lambda\right\} .
$$

Proof. Assume that $h_{M} \in\{1, \ldots, N\}$ and set $\tilde{f}=f \circ \xi^{-1}$. Choose $n_{0}>\sup _{x \in[0, D]}|\tilde{f}(x)|$ and decompose the original product into

$$
\prod_{k=1}^{N} \frac{k-h_{M}+f\left(\lambda_{k}\right)}{k-h_{M}+f\left(\lambda_{h}\right)}=\prod_{\left|k-h_{M}\right|<n_{0}} \frac{k-h_{M}+\widetilde{f}\left(\frac{k}{M}\right)}{k-h_{M}+\widetilde{f}\left(\frac{h_{M}}{M}\right)} \prod_{\left|k-h_{M}\right| \geq n_{0}} \frac{k-h_{M}+\widetilde{f}\left(\frac{k}{M}\right)}{k-h_{M}+\widetilde{f}\left(\frac{h_{M}}{M}\right)} .
$$

The first product contains a fixed finite number of factors. Each of them goes to 1 in the limit considered. Hence, the first product goes to 1 . The second can be calculated by using Lemma A.2. We obtain

$$
\lim _{\substack{N, M \rightarrow \infty \\ N / M \rightarrow D}} \prod_{k=1}^{N} \frac{k-h_{M}+f\left(\lambda_{k}\right)}{k-h_{M}+f\left(\lambda_{h_{M}}\right)}=\exp \left\{\int_{0}^{D} \frac{\widetilde{f}(x)-\widetilde{f}\left(x_{h}\right)}{x-x_{h}} d x\right\},
$$

where $x_{h}=\lim _{N, M \rightarrow+\infty} h_{M} / M$. Equation (A.8) follows from (A.10) after the change of variable $\lambda=\xi^{-1}(x)$. The case of $h_{M} \notin\{1, \ldots, N\}$ is proven along the same lines. 


\section{B The Fredholm determinant representations for the scalar products}

The proof of (4.5), (4.6) is based on the following representation for a scalar product [24, 25, 10], valid whenever $\mu_{\ell_{1}}, \ldots, \mu_{\ell_{N_{\kappa}}}$ satisfy the system (2.12) and $\nu_{1}, \ldots, \nu_{N_{\kappa}}$ are generic complex numbers:

$$
\left\langle\psi_{\kappa}(\{\mu\}) \mid \psi(\{\nu\})\right\rangle=\frac{\prod_{a=1}^{N_{\kappa}} d\left(\mu_{\ell_{a}}\right)}{\prod_{a>b}^{N_{\kappa}} \sinh \left(\mu_{\ell_{a}}-\mu_{\ell_{b}}\right) \sinh \left(\nu_{b}-\nu_{a}\right)} \cdot \operatorname{det}_{N_{\kappa}} \Omega_{\kappa}(\{\mu\},\{\nu\} \mid\{\mu\}) .
$$

The $N_{\kappa} \times N_{\kappa}$ matrix $\Omega_{\kappa}(\{\mu\},\{\nu\} \mid\{\mu\})$ is defined as

$$
\begin{aligned}
\left(\Omega_{\kappa}\right)_{j k}(\{\mu\},\{\nu\} \mid\{\mu\})=a\left(\nu_{j}\right) t\left(\mu_{\ell_{k}}, \nu_{j}\right) & \prod_{a=1}^{N_{\kappa}} \sinh \left(\mu_{\ell_{a}}-\nu_{j}-i \zeta\right) \\
& \quad-\kappa d\left(\nu_{j}\right) t\left(\nu_{j}, \mu_{\ell_{k}}\right) \prod_{a=1}^{N_{\kappa}} \sinh \left(\mu_{\ell_{a}}-\nu_{j}+i \zeta\right),
\end{aligned}
$$

with

$$
t(\mu, \nu)=\frac{-i \sin \zeta}{\sinh (\mu-\nu) \sinh (\mu-\nu-i \zeta)} \quad \text { and } \quad\left\{\begin{array}{l}
a(\mu)=\sinh ^{M}(\mu-i \zeta / 2) \\
d(\mu)=\sinh ^{M}(\mu+i \zeta / 2)
\end{array} .\right.
$$

In order to obtain the scalar product (4.5) one should set here $\nu_{j}=\lambda_{j}$ for $j=1, \ldots, N$, where $\lambda_{j}$ are the Bethe roots describing the ground state. For the scalar product (4.6) one should set in addition $\nu_{N+1}=-i \zeta / 2$.

To obtain a Fredholm determinant representation for the scalar products we should present the original determinant of the matrix $\Omega_{\kappa}$ in the following form

$$
\left.\operatorname{det}_{N_{\kappa}} \Omega_{\kappa}(\{\mu\},\{\nu\} \mid\{\mu\})\right|_{\nu_{j}=\lambda_{j}}=H(\{\mu\},\{\lambda\}) \operatorname{det}_{N}\left(\delta_{j k}+\widetilde{\Omega}\left(\lambda_{j}, \lambda_{k} \mid\{\mu\}\right) \prod_{\substack{a=1, a \neq j}}^{N}\left(\lambda_{j}-\lambda_{a}\right)^{-1}\right) .
$$

Here $\widetilde{\Omega}\left(\lambda_{j}, \lambda_{k} \mid\{\mu\}\right)$ is a new $N \times N$ matrix, $H(\{\mu\},\{\lambda\})$ an external coefficient. If we succeed to find a representation of the type (B. $\bar{B}$ ), then we can replace the determinant of the $N \times N$ matrix by Fredholm determinant of an integral operator as

$$
\operatorname{det}_{N}\left(\delta_{j k}+\widetilde{\Omega}\left(\lambda_{j}, \lambda_{k} \mid\{\mu\}\right) \prod_{\substack{a=1, a \neq j}}^{N}\left(\lambda_{j}-\lambda_{a}\right)^{-1}\right)=\operatorname{det}_{\Gamma_{q}}\left(I+\frac{1}{2 \pi i} \widetilde{\Omega}\left(w, w^{\prime} \mid\{\mu\}\right) \prod_{a=1}^{N}\left(w-\lambda_{a}\right)^{-1}\right) .
$$

Here the integral operator in the r.h.s. of (B.5) acts on a counterclockwise oriented contour $\Gamma_{q}$ surrounding the Fermi zone $[-q, q]$. This contour is such that it contains all the ground state roots $\lambda_{j}$ and no other singularity of the kernel $\widetilde{\Omega}\left(w, w^{\prime} \mid\{\mu\}\right)$. 
The proof of (B.5) is quite obvious. Indeed, expanding the Fredholm determinant into the series of multiple integrals we see that each of these integrals reduces to the sum of the residues in the points $\lambda_{j}$. Thus, the series of multiple integrals turns into the series of multiple sums. Then one can easily convince oneself that this series coincides with the expansion of the $N \times N$ determinant in the l.h.s. of (B.5).

Thus, our goal is to pass from the original representation (B.1) to the form (B.4 $)$. The way we use here is based on the extraction of a Cauchy determinant from the original determinant defining the scalar product, an idea which was first introduced in [55, 56]. In the case of the scalar product (4.5) this was done in [36]. The same method with minor modifications described below can be used for the proof of the second determinant identity (4.6).

Following [36], we consider the matrix $\Omega_{\kappa}(\{z\},\{\nu\} \mid\{z\})$ for two sets of $N_{\kappa}=N+1$ generic parameters $\{z\}$ and $\{\nu\}$. In this case we write

$$
\operatorname{det}_{N+1}\left[\Omega_{\kappa}\right]=\frac{\operatorname{det}_{N+1}\left(\Omega_{\kappa} A\right)}{\operatorname{det}_{N+1} A} \quad \text { with } A_{j k}=\frac{\prod_{a=1}^{N+1} \sinh \left(z_{j}-\nu_{a}\right)}{\prod_{\substack{a=1 \\ a \neq j}}^{N+1} \sinh \left(z_{j}-z_{a}\right)} \times \begin{cases}\operatorname{coth}\left(z_{j}-\nu_{k}\right) & \text { for } k \leq N, \\ 1 & \text { for } k=N+1 .\end{cases}
$$

The effect of multiplication by the matrix $A$ is computed similarly to the method presented in [36]. We get

$$
\operatorname{det}_{N+1}\left[\Omega_{\kappa}(\{z\},\{\nu\} \mid\{z\})\right]=\operatorname{det}_{N+1}\left[\frac{1}{\sinh \left(z_{k}-\nu_{j}\right)}\right] \cdot \operatorname{det}_{N+1} S_{j k}
$$

where

$$
\begin{gathered}
S_{j k}=\delta_{j k} \mathcal{Y}_{\kappa}\left(\nu_{j} \mid\{z\}\right)+\left.\frac{\prod_{a=1}^{N+1} \sinh \left(\nu_{k}-z_{a}\right)}{\prod_{\substack{a=1 \\
a \neq k}}^{N+1} \sinh \left(\nu_{k}-\nu_{a}\right)} \cdot \frac{\partial}{\partial y_{k}} \mathcal{Y}_{\kappa}\left(\nu_{j} \mid\{y\}\right)\right|_{\{y\}=\{\nu\}}, \quad k \leq N, \\
S_{j, N+1}=\mathcal{Y}_{\kappa}\left(\nu_{j} \mid\{\nu\}\right),
\end{gathered}
$$

and we have set for arbitrary complex $y_{1}, \ldots, y_{N+1}$

$$
\mathcal{Y}_{\kappa}(\nu \mid\{y\})=a(\nu) \prod_{k=1}^{N+1} \sinh \left(y_{k}-\nu-i \zeta\right)+\kappa d(\nu) \prod_{k=1}^{N+1} \sinh \left(y_{k}-\nu+i \zeta\right)
$$

Finally we reduce the size of $\operatorname{det}_{N+1} S$ by one by performing linear combinations of the lines

$$
\operatorname{det}_{N+1}\left[S_{j k}\right]=S_{N+1, N+1} \operatorname{det}_{N}\left[S_{j k}-S_{j, N+1} \frac{S_{N+1, k}}{S_{N+1, N+1}}\right] .
$$

Thus, we arrive at the following representation for $\operatorname{det}_{N+1} \Omega_{\kappa}$ depending on generic complex $z_{1}, \ldots, z_{N+1}$ and $\nu_{1}, \ldots, \nu_{N+1}$ :

$$
\underset{N+1}{\operatorname{det}}\left[\Omega_{\kappa}(\{z\},\{\nu\} \mid\{z\})\right]=S_{N+1, N+1} \operatorname{det}_{N+1}\left[\frac{1}{\sinh \left(z_{k}-\nu_{j}\right)}\right] \underset{N}{\operatorname{det}}\left[S_{j k}-S_{j, N+1} \frac{S_{N+1, k}}{S_{N+1, N+1}}\right],
$$

where $S_{j k}$ are given by (B.8), (B.9). 
Now we set $z_{a}=\left\{\mu_{\ell_{a}}\right\}$ for $a=1, \ldots, N+1, \nu_{k}=\lambda_{k}$ for $k=1, \ldots, N$ and $\nu_{N+1}=-i \zeta / 2$. We assume that the parameters $\left\{\mu_{\ell_{a}}\right\}$ satisfy the system (2.11), while the parameters $\left\{\lambda_{k}\right\}$ satisfy the system (2.2). Then we obtain for $j \leq N$,

$$
\begin{aligned}
& \frac{\mathcal{Y}_{\kappa}\left(\nu_{j} \mid\{\nu\}\right)}{a\left(\lambda_{j}\right) \prod_{b=1}^{N} \sinh \left(\lambda_{b}-\lambda_{j}-i \zeta\right)}=\kappa \sinh \left(\lambda_{j}-\frac{i \zeta}{2}\right)-\sinh \left(\lambda_{j}+\frac{3 i \zeta}{2}\right) \\
& \left.\frac{\partial \mathcal{Y}_{\kappa}\left(\nu_{j} \mid\{y\}\right) / \partial y_{k}}{a\left(\lambda_{j}\right) \prod_{b=1}^{N} \sinh \left(\lambda_{b}-\lambda_{j}-i \zeta\right)}\right|_{\{y\}=\{\nu\}}=\kappa \frac{\sinh \left(\lambda_{j}-\frac{i \zeta}{2}\right)}{\tanh \left(\lambda_{k}-\lambda_{j}+i \zeta\right)}-\frac{\sinh \left(\lambda_{j}+\frac{3 i \zeta}{2}\right)}{\tanh \left(\lambda_{k}-\lambda_{j}-i \zeta\right)},
\end{aligned}
$$

and for $j=N+1$,

$$
\begin{aligned}
& \mathcal{Y}_{\kappa}\left(\nu_{N+1} \mid\{\nu\}\right)=a(-i \zeta / 2) \sinh (-i \zeta) \prod_{b=1}^{N} \sinh \left(\lambda_{b}-\frac{i \zeta}{2}\right), \\
& \partial \mathcal{Y}_{\kappa}\left(\nu_{N+1} \mid\{y\}\right) /\left.\partial y_{k}\right|_{\{y\}=\{\nu\}}=a(-i \zeta / 2) \sinh (-i \zeta) \operatorname{coth}\left(\lambda_{k}-\frac{i \zeta}{2}\right) \prod_{b=1}^{N} \sinh \left(\lambda_{b}-\frac{i \zeta}{2}\right) .
\end{aligned}
$$

It also follows from (2.4), (2.12) and the definition of the shift function (2.13) that

$$
\kappa \prod_{a=1}^{N+1} \frac{\sinh \left(\mu_{\ell_{a}}-w+i \zeta\right)}{\sinh \left(\mu_{\ell_{a}}-w-i \zeta\right)} \prod_{a=1}^{N} \frac{\sinh \left(\lambda_{a}-w-i \zeta\right)}{\sinh \left(\lambda_{a}-w+i \zeta\right)}=e^{2 \pi i \widehat{F}(w)}
$$

Substituting all these formulae into (B.12) we arrive after simple algebra at the representation of the form (B.4), and thus, we obtain the Fredholm determinant representation (4.6).

\section{References}

[1] N. Kitanine, K. K. Kozlowski, J. M. Maillet, N. A. Slavnov and V. Terras, J. Math. Phys. 50 (2009) 095209.

[2] W. Heisenberg, Zeitschrift für Physik, 49 (1928) 619.

[3] H. Bethe, Zeitschrift für Physik, 71 (1931) 205.

[4] L. Hulthen, Arkiv. Mat. Astron. Fys. A 26 (1938) 1.

[5] R. Orbach, Phys. Rev. 112 (1958) 309.

[6] L. R. Walker, Phys. Rev. 116 (1959) 1089.

[7] J. Des Cloizeaux and M. Gaudin, J. Math. Phys. 7 (1966) 1384.

[8] C. N. Yang and C. P. Yang, Phys. Rev. 150, (1966), 321.

[9] E. Lieb and D. Mattis (eds.), Mathematical Physics in One Dimension, New York: Academic Press, (1966).

[10] N. Kitanine, J. M. Maillet and V. Terras, Nucl. Phys. B 554 [FS] (1999) 647. 
[11] L. D. Faddeev, E. K. Sklyanin and L. A. Takhtajan, Theor. Math. Phys. 40 (1980) 688.

[12] L.D. Faddeev, in: Les Houches Lectures Quantum Symmetries, eds A. Connes et al, (North Holland 1998), 149.

[13] V. E. Korepin, N. M. Bogoliubov, A. G. Izergin, Quantum Inverse Scattering Method and Correlation Functions, Cambridge University Press, 1993.

[14] J. S. Caux and J. M. Maillet, Phys. Rev. Lett. 95 (2005) 077201.

[15] J. S. Caux, R. Hagemans, and J. M. Maillet, J. Stat. Mech. Theory Exp. (2005) P09003.

[16] R. G. Pereira, J. Sirker J, J. S. Caux, R. Hagemans, J. M. Maillet, S. R. White and I. Affleck, Phys. Rev. Lett. 96 (2006) 257202.

[17] R. G. Pereira, J. Sirker J, J. S. Caux, R. Hagemans, J. M. Maillet, S. R. White and I. Affleck, J. Stat. Mech. Theory Exp. (2007) P08022.

[18] J. S. Caux, P. Calabrese and N. A. Slavnov, J. Stat. Mech. Theory Exp. (2007) P01008.

[19] T. Kojima, V.E. Korepin and N.A. Slavnov, Comm. Math. Phys. 188 (1997) 657.

[20] V. E. Korepin and N. A. Slavnov, Int. J. Mod. Phys. B 13 (1999) 2933.

[21] O. A. Castro-Alvaredo and J. M. Maillet, J. Phys. A: Math. Gen. 40 (2007) 7451.

[22] T. Deguchi and C. Matsui, Nucl. Phys. B 814 (2009) 409.

[23] J. M. Maillet and V. Terras, Nucl. Phys. B 575 (2000) 627.

[24] N. A. Slavnov, Theor. Math. Phys. 79 (1989) 502.

[25] N. A. Slavnov, Zap. Nauchn. Sem. POMI 245 (1997) 270.

[26] N. A. Slavnov, Russian Mathematical Surveys 62 (2007) 727.

[27] N. Kitanine, J. M. Maillet, N. A. Slavnov and V. Terras, Nucl. Phys. B 712 (2005) 600.

[28] N. Kitanine, J. M. Maillet, N. A. Slavnov and V. Terras, Nucl. Phys. B 729 (2005) 558.

[29] N. Kitanine, J. M. Maillet, N. A. Slavnov and V. Terras, in "Solvable lattice models 2004", RIMS publications, 2006, hep-th/0505006.

[30] M. Jimbo, K. Miki, T. Miwa, and A. Nakayashiki, Phys. Lett. A 168 (1992) 256.

[31] M. Jimbo and T. Miwa, Journ. Phys. A: Math. Gen. 29 (1996) 2923.

[32] M. Jimbo and T. Miwa, Algebraic analysis of solvable lattice models (AMS, 1995).

[33] N. Kitanine, J. M. Maillet, and V. Terras, Nucl. Phys. B 567 (2000) 554.

[34] N. Kitanine, J. M. Maillet, N. A. Slavnov and V. Terras, Nucl. Phys. B 641 [FS] (2002) 487. 
[35] N. Kitanine, J. M. Maillet, N. A. Slavnov, and V. Terras, Nucl. Phys. B 642 (2002) 433.

[36] N. Kitanine, K. K. Kozlowski, J. M. Maillet, N. A. Slavnov and V. Terras, J. Stat. Mech. (2009) P04003.

[37] A. Luther and I. Peschel, Phys. Rev. B 12 (1975) 3908.

[38] F. D. M. Haldane, Phys. Rev. Lett. 45 (1980) 1358.

[39] F. D. M. Haldane, Phys. Lett. A 81 (1981) 153.

[40] F. D. M. Haldane, J. Phys. C: Solid State Phys. 14 (1981) 2585.

[41] I. Affleck, Phys. Rev. Lett. 55 (1985) 1355.

[42] H. W. J. Blöte, J. L. Cardy and M. P. Nightingale, Phys. Rev. Lett. 56 (1986) 742.

[43] J. L. Cardy, J. Phys. A: Math. Gen. 17 (1984) L385.

[44] J. L. Cardy, Nucl. Phys. B 270 (1986) 186.

[45] E. Lieb, T. Schultz, and D. Mattis, Ann. Phys. 16 (1961) 407.

[46] V. E. Korepin and N. A. Slavnov, Eur. Phys. J. B 5 (1998) 555.

[47] N. A. Slavnov, Theor. Math. Phys. 116 (1998) 362.

[48] O. Babelon, H. J. de Vega, and C.-M. Viallet, Nucl. Phys. B 220 (1983) 13.

[49] S. Lukyanov, 1999 Phys. Rev. B 59 (1999) 11163.

[50] S. Lukyanov and V. Terras, Nucl. Phys. B 654 (2003) 323.

[51] M. Gaudin, B. M. McCoy and T. W. Tai, Phys. Rev. D 23 (1981) 417.

[52] V. E. Korepin, Comm. Math. Phys. 86 (1982) 391.

[53] M. Gaudin, La fonction d'onde de Bethe, Masson, 1983.

[54] N. Kitanine, K. K. Kozlowski, J. M. Maillet, N. A. Slavnov and V. Terras, Form factor approach to the asymptotic behavior of correlation functions in critical models, to appear.

[55] N. A. Slavnov, Theor. Math. Phys. 82 (1990) 273.

[56] A. G. Izergin, N. Kitanine, J. M. Maillet and V. Terras, Nucl. Phys. B 554 [FS] (1999) 679. 